ESAIM: M2AN 50 (2016) 337-360

DOI: $10.1051 / \mathrm{m} 2 \mathrm{an} / 2015045$
ESAIM: Mathematical Modelling and Numerical Analysis

www.esaim-m2an.org

\title{
A DISCONTINUOUS GALERKIN REDUCED BASIS ELEMENT METHOD FOR ELLIPTIC PROBLEMS
}

\author{
Paola F. Antonietti ${ }^{1}$, Paolo Pacciarini ${ }^{1}$ and Alfio Quarteroni ${ }^{1,2}$
}

\begin{abstract}
We propose and analyse a new discontinuous reduced basis element method for the approximation of parametrized elliptic PDEs in partitioned domains. The method is built upon an offline stage (parameter independent) and an online (parameter dependent) one. In the offline stage we build a non-conforming (discontinuous) global reduced space as a direct sum of local basis functions generated independently on each subdomain. In the online stage, for any given value of the parameter, the approximate solution is obtained by ensuring the weak continuity of the fluxes and of the solution itself thanks to a discontinuous Galerkin approach. The new method extends and generalizes the methods introduced in [L. Iapichino, Ph.D. thesis, EPF Lausanne (2012); L. Iapichino, A. Quarteroni and G. Rozza, Comput. Methods Appl. Mech. Eng. 221-222 (2012) 63-82]. We prove its stability and convergence properties, as well as the spectral properties of the associated online algebraic system. We also propose a two-level preconditioner for the online problem which exploits the pre-existing decomposition of the domain and is based upon the introduction of a global coarse finite element space. Numerical tests are performed to verify our theoretical results.
\end{abstract}

Mathematics Subject Classification. 65N12, 65N30.

Received August 30, 2014. Revised June 17, 2015.

Published online February 16, 2016.

\section{INTRODUCTION}

The Reduced Basis (RB) method, see e.g. [32-34], for elliptic Parametrized Partial Differential Equations (PPDEs) has been successfully developed to approximate the solution of problems like:

$$
\text { find } u(\boldsymbol{\mu}) \in V \text { such that } A(u(\boldsymbol{\mu}), v ; \boldsymbol{\mu})=F(v ; \boldsymbol{\mu}) \quad \forall v \in V,
$$

where $V$ is a suitable Hilbert space, $\boldsymbol{\mu}=\left(\mu_{1}, \ldots, \mu_{P}\right)$ is a $P$-tuple of parameters which belongs to a subspace $\mathcal{D}$ of $\mathbb{R}^{P}, A$ is a continuous coercive bilinear form defined on $V \times V$ and $F$ is a linear continuous functional on $V$.

When the domain $\Omega(\boldsymbol{\mu})$ of the PPDE is partitioned into several subdomains, a convenient numerical approach is provided by the so-called Reduced Basis Element (RBE) method, presented in [26-29], in which local (i.e.,

\footnotetext{
Keywords and phrases. Reduced basis element method, discontinuous Galerkin, domain decomposition.

1 MOX-Modeling and Scientific Computing, Dipartimento di Matematica, Politecnico di Milano, Piazza Leonardo da Vinci 32, 20133 Milano, Italy. paola.antonietti@polimi.it paolo.pacciarini@polimi.it alfio.quarteroni@polimi.it

2 CMCS, École Polytechnique Fédérale de Lausanne (EPFL), Station 8, 1015 Lausanne, Switzerland.

alfio.quarteroni@epfl.ch
} 
defined on each subdomain) reduced bases are built by restriction of global solutions, while the global continuity of the RB solution is guaranteed either by the introduction of suitable Lagrange multipliers, as in [24], or by adopting a discontinuous Galerkin (DG) approach, as in [13].

Several improvements of the Reduced Basis Element (RBE) idea have been recently proposed. One instance is the so-called static condensation Reduced Basis Element method [15,19,20], where a RB approximation of the Schur complement is proposed and rigorous a posteriori error estimators are derived. Another approach is represented by the so-called Reduced Basis Hybrid Method (RBHM) [22, 24], where a global coarse solution, responsible for ensuring interface continuity of normal fluxes, is overlaid to the subspace of local reduced basis computed offline at subdomain levels. The continuity of the global reduced solution is enforced using Lagrange multipliers. A further instance is provided by the Reduced Basis-Domain Decomposition-Finite Element (RDF) method [22,23], in which the continuity of the elements of the reduced space on the whole domain $\Omega(\boldsymbol{\mu})$ is guaranteed by the introduction of additional degrees of freedom on the interfaces, corresponding to the fine-grid Finite Element (FE) Lagrangian basis functions associated with the nodes on each interface. Ideas related to the RBE approach can also be applied to the RB approximation of multiscale phenomena, as done in $[1,25]$. Besides the RB framework, a method that shows similarities with the RBE approach is the Generalized Multiscale Finite Element Method presented in [14,17]. In the latter, the DG approach is employed to impose weak continuity of the global solution, which belongs to a discontinuous space spanned by local bases

computed subdomainwise. However, differently from the approach proposed here, the local spaces are not built with a Greedy algorithm but solving a number of local eigenvalue problems.

In this work we propose a discontinuous Galerkin Reduced Basis Element (DGRBE) method which represents in fact a generalization and an improvement of both RDF and RBHM. As a matter of fact, as in the RDF method, the DGRBE approximation is based upon a set of local basis functions that feature non-homogeneous Neumann boundary conditions, without however requiring the introduction of additional degrees of freedom on the interfaces. Moreover, a possible preconditioner for the reduced problem is introduced by making use of a coarse space correction on the local basis inspired by the RBHM. We point out that this correction was essential to ensure interface stress continuities in RBHM, whereas in our DGRBE method it only serves the purpose of improving the spectral properties of the preconditioner to solve the associated online linear system.

Furthermore, the underlying DG approach allows for the use of independent elementwise representation of the numerical solution, without necessitating Lagrange multipliers to ensure the continuity across the internal interfaces, as was the case for RBHM. The DGRBE method is then well suited for global meshes which are non-conforming on the subdomain interfaces. We point out that the local bases are constructed by solving local problems with suitably chosen boundary conditions. No approximate solution of the global problem (1.1) is therefore required. This makes the DGRBE method particularly well suited for problems defined on "modular" domains, namely composed by an arbitrary number of subdomains that can be obtained by geometrical transformation of few parameter-independent reference subdomains, $c f$. [19,24]. We show by numerical experiments that the DGRBE approximation of (1.1) on a partitioned domain is as accurate as a fine-grid FE one, even though it is based on a significantly lower dimensional approximation space. After introducing the DGRBE method, we carry out its analysis in the case of elliptic problems. More precisely, we prove: the well-posedness of the method, its stability and some convergence estimates.

An outline of the paper is as follows. In Section 2 the main features of the DGRBE method are introduced, while in Section 3 the theoretical analysis is carried out. In Section 4 a two level preconditioner is presented and it is meant to make the preconditioned online system weakly scalable. Finally, in Section 5 some numerical tests are shown. In the appendices some implementation details are reported.

\section{The DGRBE METHOD}

We assume that a parameter dependent open subset $\Omega(\boldsymbol{\mu}) \subset \mathbb{R}^{2}$ is given, where $\boldsymbol{\mu}$ is a parameter belonging to the space $\mathcal{D} \subseteq \mathbb{R}^{P}, P \geq 1$. Given an integer $N_{S}>1$, we assume that the domain is composed of a finite 
number of non-overlapping subdomains,

$$
\bar{\Omega}(\boldsymbol{\mu})=\bigcup_{i=1}^{N_{S}} \bar{\Omega}_{i}(\boldsymbol{\mu}) \quad \forall \boldsymbol{\mu} \in \mathcal{D}
$$

where each $\Omega_{i}(\boldsymbol{\mu})$ is an open bounded subset of $\mathbb{R}^{2}$. The model problem we are considering is the following:

$$
\begin{aligned}
-\nu(\boldsymbol{\mu}) \Delta u(\boldsymbol{\mu})+\sigma(\boldsymbol{\mu}) u(\boldsymbol{\mu}) & =f(\boldsymbol{\mu}) \text { in } \Omega(\boldsymbol{\mu}), \\
u(\boldsymbol{\mu}) & =0 \quad \text { on } \partial \Omega(\boldsymbol{\mu}),
\end{aligned}
$$

where $f \in \mathrm{L}^{2}(\Omega(\boldsymbol{\mu}))$ is a given source term and $\nu(\boldsymbol{\mu}), \sigma(\boldsymbol{\mu})$ are $\boldsymbol{\mu}$-dependent constant coefficients. We point out that our results can be extended to the case of subdomainwise constant coefficients or, under suitable regularity assumptions, to the case of space-dependent functions. We also remark that the parameter dependence can be both physical and geometrical, that is both the coefficients and the domain can depend on the parameters. To follow a reduced basis approach, we define a reference domain $\Omega=\Omega(\overline{\boldsymbol{\mu}})$, for a suitably chosen $\overline{\boldsymbol{\mu}} \in \mathcal{D}$. Correspondingly, we define the reference subdomains $\Omega_{i}=\Omega_{i}(\overline{\boldsymbol{\mu}})$, for $i=1 \ldots N_{S}$. Let $T_{i}^{\mu}: \Omega_{i} \rightarrow \Omega_{i}(\boldsymbol{\mu})$, be the local geometrical transformation mapping the reference subdomains into the "physical" ones. By patching together these local transformations, we can define a global transformation $T^{\mu}$ which maps the reference domain $\Omega$ onto $\Omega(\boldsymbol{\mu})$. We assume that the global map $T^{\mu}$ is continuous and bijective. Setting $V=\mathrm{H}_{0}^{1}(\Omega)$, we define

$$
A(w, v ; \boldsymbol{\mu})=\int_{\Omega(\boldsymbol{\mu})} \nu(\boldsymbol{\mu}) \nabla\left(w \circ\left(T^{\boldsymbol{\mu}}\right)^{-1}\right) \cdot \nabla\left(v \circ\left(T^{\boldsymbol{\mu}}\right)^{-1}\right) \mathrm{d} x+\int_{\Omega(\boldsymbol{\mu})} \sigma(\boldsymbol{\mu})\left(w \circ\left(T^{\boldsymbol{\mu}}\right)^{-1}\right)\left(v \circ\left(T^{\boldsymbol{\mu}}\right)^{-1}\right) \mathrm{d} x,
$$

for all $w, v \in V$. For the sake of notation, in what follows we let the composition with $\left(T^{\mu}\right)^{-1}$ to be understood.

The reduced-order method we are going to introduce features two main components:

- a local reduced basis for each subdomain;

- a DG-type interface treatment at subdomain boundaries.

In what follows we explain the role played by these components during the two stages of the DGRBE method: the offline stage and the online stage. Details about the implementation aspects can be found in Appendix A.

\subsection{Offline stage}

The offline stage of the DGRBE method is inspired by the offline stage of the RDF method introduced in [22] and follows ideas which can also be found in [19]. For each $i=1, \ldots, N_{S}$, we define a (parameter independent) conforming quasi-uniform triangulation $\mathcal{T}_{h, i}$ on $\Omega_{i}$. Setting $\Gamma_{i}=\partial \Omega_{i} \backslash \partial \Omega$, we define the local spaces

$$
\begin{aligned}
& V_{i}=\left\{v_{i} \in \mathrm{L}^{2}(\Omega)\left|v_{i}\right|_{\Omega_{i}} \in \mathrm{H}^{1}\left(\Omega_{i}\right), v_{i}=0 \text { on } \partial \Omega_{i} \backslash \Gamma_{i}, v_{i}=0 \text { in } \Omega \backslash \Omega_{i}\right\}, \\
& V_{h, i}=\left\{v_{h, i} \in V_{i}\left|v_{h, i}\right|_{K} \in \mathbb{P}^{1}(K) \forall K \in \mathcal{T}_{h, i}\right\} .
\end{aligned}
$$

On each subdomain we build a local reduced basis such that, for each value of the parameters, it allows a good approximation of the solution of the two following problems:

$$
\begin{aligned}
-\nu(\boldsymbol{\mu}) \Delta \dot{\mathrm{i}}_{i}(\boldsymbol{\mu})+\sigma(\boldsymbol{\mu}) \dot{\mathrm{i}}_{i}(\boldsymbol{\mu}) & =f(\boldsymbol{\mu}), & & \text { in } \Omega_{i}(\boldsymbol{\mu}) \\
\frac{\partial \dot{u}_{i}(\boldsymbol{\mu})}{\partial n_{i}} & =0, & & \text { on } \Gamma_{i}(\boldsymbol{\mu}) \\
\stackrel{\mathrm{u}}{i}_{i}(\boldsymbol{\mu}) & =0, & & \text { on } \partial \Omega_{i}(\boldsymbol{\mu}) \backslash \Gamma_{i}(\boldsymbol{\mu}),
\end{aligned}
$$

and

$$
\begin{aligned}
-\nu(\boldsymbol{\mu}) \Delta w_{i}(\boldsymbol{\mu}, \beta)+\sigma(\boldsymbol{\mu}) w_{i}(\boldsymbol{\mu}, \beta) & =0, \quad \text { in } \Omega_{i}(\boldsymbol{\mu}) \\
\frac{\partial w_{i}(\boldsymbol{\mu}, \beta)}{\partial n_{i}} & =g(\beta), \text { on } \Gamma_{i}(\boldsymbol{\mu}) \\
w_{i}(\boldsymbol{\mu}, \beta) & =0, \quad \text { on } \partial \Omega_{i}(\boldsymbol{\mu}) \backslash \Gamma_{i}(\boldsymbol{\mu}),
\end{aligned}
$$


where $g(\beta)$ is a Neumann datum which depends on an additional parameter $\beta \in \mathbb{N}$. Recalling that $u(\boldsymbol{\mu})$ is the solution of (2.1), we now observe that if, for a given set of parameters $\left\{\beta_{1}, \ldots, \beta_{\tilde{n}}\right\} \subset \mathbb{N}$ all referring to the current subdomain $\Omega_{i}$ (we avoid indexing $\beta_{j}$ as $\beta_{j}^{i}, j=1, \ldots, \widetilde{n}$ for the sake of notation), $\sum_{j} g\left(\beta_{j}\right)$ is a good approximation of $\left.\frac{\partial u(\boldsymbol{\mu})}{\partial n}\right|_{\Gamma_{i}}$ then by linearity $u_{i}(\boldsymbol{\mu})=\stackrel{\mathrm{u}}{i}_{i}(\boldsymbol{\mu})+\sum_{j} w_{i}\left(\boldsymbol{\mu}, \beta_{j}\right)$ will be a good approximation of $\left.u(\boldsymbol{\mu})\right|_{\Omega_{i}(\boldsymbol{\mu})}$. Thus, building a space able to approximate the solutions of problems (2.2) and (2.3) on each subdomain allows to approximate also the solution of the initial problem (2.1). In the following we make the above idea more clear. We first introduce the local forms

$$
A_{i}\left(w_{i}, v_{i} ; \boldsymbol{\mu}\right)=\int_{\Omega_{i}(\boldsymbol{\mu})} \nu(\boldsymbol{\mu}) \nabla w_{i} \cdot \nabla v_{i} \mathrm{~d} x+\int_{\Omega_{i}(\boldsymbol{\mu})} \sigma(\boldsymbol{\mu}) w_{i} v_{i} \mathrm{~d} x, \quad F_{i}\left(v_{i} ; \boldsymbol{\mu}\right)=\int_{\Omega_{i}(\boldsymbol{\mu})} f(\boldsymbol{\mu}) v_{i} \mathrm{~d} x,
$$

for all $w_{i}, v_{i} \in V_{i}$. We then define

$$
\left(w_{i}, v_{i}\right)_{V_{i}}=A_{i}\left(w_{i}, v_{i} ; \overline{\boldsymbol{\mu}}\right), \quad\left\|v_{i}\right\|_{V_{i}}=\left(v_{i}, v_{i}\right)_{V_{i}}^{1 / 2} \quad \forall w_{i}, v_{i} \in V_{i}
$$

where $\overline{\boldsymbol{\mu}}$ is the parameter value chosen to identify the reference domain. Next, we define the extended parameter space

$$
\widetilde{\mathcal{D}}=\mathcal{D} \times\left\{0, \ldots, n_{B C, i}\right\}, \quad \text { with } n_{B C, i} \in \mathbb{N},
$$

and we denote with $\widetilde{\boldsymbol{\mu}}=(\boldsymbol{\mu}, \beta)$ the generic element of $\widetilde{\mathcal{D}}$. Denoting with $V_{h, i}^{\Gamma_{i}}$ the space of the traces on $\Gamma_{i}$ of the elements of $V_{h, i}$, we introduce a $\beta$-dependent functional $\mathcal{I}_{i}^{\beta}$ belonging to the dual space of $V_{h, i}^{\Gamma_{i}}$, for all $\beta \in\left\{0, \ldots, n_{B C, i}\right\}$. We are now able to define the local problems which we use to build the local basis. Given a parameter value $\widetilde{\boldsymbol{\mu}} \in \widetilde{\mathcal{D}}$, find $\widetilde{u}_{h, i}(\widetilde{\boldsymbol{\mu}}) \in V_{h, i}$ such that

$$
A_{i}\left(\widetilde{u}_{h, i}(\widetilde{\boldsymbol{\mu}}), v_{h, i} ; \widetilde{\boldsymbol{\mu}}\right)=\widetilde{F}_{i}\left(v_{h, i} ; \tilde{\boldsymbol{\mu}}\right)+\left\langle\mathcal{I}_{i}^{\beta},\left.v_{h, i}\right|_{\Gamma_{i}}\right\rangle \quad \forall v_{h, i} \in V_{h, i},
$$

We assume that, for each choice of $w_{h, i}$ and $v_{h, i}$ in $V_{h, i}$, it holds

$$
\begin{aligned}
& \widetilde{F}_{i}\left(v_{h, i} ; \widetilde{\boldsymbol{\mu}}\right)=F_{i}\left(v_{h, i} ; \boldsymbol{\mu}\right) \quad \text { and } \quad\left\langle\mathcal{I}_{i}^{\beta},\left.v_{h, i}\right|_{\Gamma_{i}}\right\rangle=0 \quad \forall \widetilde{\boldsymbol{\mu}} \in \mathcal{D} \times\{0\}, \text { i.e., when } \beta=0, \\
& \widetilde{F}_{i}\left(v_{h, i} ; \tilde{\boldsymbol{\mu}}\right)=0 \quad \forall \widetilde{\boldsymbol{\mu}} \in \mathcal{D} \times\left\{1, \ldots, n_{B C, i}\right\}
\end{aligned}
$$

We observe that we are considering the FE approximation of problems (2.2) and (2.3) when $\beta=0$ and $\beta \neq 0$, respectively. The linear functional $\mathcal{I}_{i}^{\beta}$ serves the purpose of (weakly) imposing the non-homogeneous Neumann boundary conditions. We propose two possible choices of $\mathcal{I}_{i}^{\beta}$ : the former is based on the approximation of the weak normal derivative of the fine FE solution, the latter on the approximation of the normal derivative of the continuous global solution along the internal interfaces.

The well-posedness of the local problem (2.5) is guaranteed by the following lemma, which can be proven using a standard energy argument (cf. [31]).

Lemma 2.1. Let $D_{i}^{\mu}$ be the Jacobian matrix of $T_{i}^{\mu}$ and let $J_{i}^{\mu}$ be its determinant. For every $\boldsymbol{\mu}$ in $\mathcal{D}$, we assume that $\nu(\boldsymbol{\mu})>0, \sigma(\boldsymbol{\mu})>0$ and $J_{i}^{\mu}>0$ on $\Omega_{i}, i=1, \ldots, N_{S}$, and define

$$
\begin{aligned}
& \alpha_{i}(\boldsymbol{\mu})=\min \left\{\frac{\nu(\boldsymbol{\mu})}{\nu(\overline{\boldsymbol{\mu}})}, \frac{\sigma(\boldsymbol{\mu})}{\sigma(\overline{\boldsymbol{\mu}})}\right\} \min _{x \in \Omega_{i}}\left[\min \left\{\lambda_{\min }\left(\left(D_{i}^{\boldsymbol{\mu}}\right)^{-1}\left(D_{i}^{\boldsymbol{\mu}}\right)^{-\top}\right), 1\right\} J_{i}^{\boldsymbol{\mu}}\right], \\
& K_{i}(\boldsymbol{\mu})=\max \left\{\frac{\nu(\boldsymbol{\mu})}{\nu(\overline{\boldsymbol{\mu}})}, \frac{\sigma(\boldsymbol{\mu})}{\sigma(\overline{\boldsymbol{\mu}})}\right\} \max _{x \in \Omega_{i}}\left[\max \left\{\lambda_{\max }\left(\left(D_{i}^{\boldsymbol{\mu}}\right)^{-1}\left(D_{i}^{\boldsymbol{\mu}}\right)^{-\top}\right), 1\right\} J_{i}^{\boldsymbol{\mu}}\right] .
\end{aligned}
$$

Then, for $i=1, \ldots, N_{S}$, there exist two constants $\alpha_{i}(\boldsymbol{\mu}), K_{i}(\boldsymbol{\mu})$ such that:

(i) $0<\alpha_{i}(\boldsymbol{\mu})<K_{i}(\boldsymbol{\mu})$; 
(ii) for each $v_{i}, w_{i}$ in $V_{i}$ and for each $\boldsymbol{\mu}$ in $\mathcal{D}$,

$$
\alpha_{i}(\boldsymbol{\mu})\left\|v_{i}\right\|_{V_{i}}^{2} \leq A_{i}\left(v_{i}, v_{i} ; \boldsymbol{\mu}\right), \quad\left|A_{i}\left(w_{i}, v_{i} ; \boldsymbol{\mu}\right)\right| \leq K_{i}(\boldsymbol{\mu})\left\|w_{i}\right\|_{V_{i}}\left\|v_{i}\right\|_{V_{i}} .
$$

By applying the Greedy's algorithm $[32,34]$ to problem $(2.5)$ we obtain a local RB space

$$
V_{i}^{R B}=\operatorname{span}\left\{\widetilde{u}_{h, i}\left(\widetilde{\boldsymbol{\mu}}_{i}^{1}\right), \ldots, \widetilde{u}_{h, i}\left(\widetilde{\boldsymbol{\mu}}_{i}^{N_{i}}\right)\right\},
$$

for a suitably chosen set of parameters $\widetilde{\boldsymbol{\mu}}_{i}^{k}, k=1, \ldots, N_{i}$. Then the solution $u_{i}^{R B}(\widetilde{\boldsymbol{\mu}}) \in V_{i}^{R B}$ of

$$
A_{i}\left(\widetilde{u}_{i}^{R B}(\widetilde{\boldsymbol{\mu}}), v_{i}^{R B} ; \widetilde{\boldsymbol{\mu}}\right)=\widetilde{F}_{i}\left(v_{i}^{R B} ; \widetilde{\boldsymbol{\mu}}\right)+\left\langle\mathcal{I}_{i}^{\beta},\left.v_{i}^{R B}\right|_{\Gamma_{i}}\right\rangle \quad \forall v_{i}^{R B} \in V_{i}^{R B},
$$

satisfies

$$
\left\|\widetilde{u}_{h, i}(\widetilde{\boldsymbol{\mu}})-\widetilde{u}_{i}^{R B}(\widetilde{\boldsymbol{\mu}})\right\|_{\boldsymbol{\mu}} \leq \varepsilon^{*}\left\|\widetilde{u}_{i}^{R B}(\widetilde{\boldsymbol{\mu}})\right\|_{V_{i}} \quad \forall \boldsymbol{\mu} \in \Xi,
$$

for a given (small) tolerance $\varepsilon *$. Here $\Xi$ denotes a finite training subset of $\mathcal{D}$, which is needed to perform the Greedy's algorithm [34]. As usual in the RB context, we assume that $\Xi$ is sufficiently "dense" in $\mathcal{D}$. This ensures that the Greedy's algorithm is insensitive to the specific training subset that has been chosen $[30,34]$. Moreover, we observe that under suitable assumptions on the equation coefficients, such as smooth or Lipschitz dependence on the parameter $[16,30]$, inequality $(2.6)$ could be extended (even though slightly weakened) to the whole parameter set $\mathcal{D}$. The global DGRBE space is now defined as

$$
V^{R B}=\bigoplus_{i=1}^{N_{S}} V_{i}^{R B}
$$

A basis of the space $V^{R B}$ is $\mathcal{B}_{R B}=\bigcup_{i=1}^{N_{S}} \mathcal{B}_{R B, i}$.

Remark 2.2. The set of parameters on which a single local problem depend can be smaller than the global set of parameters associated with problem (2.1). For instance if the domain $\Omega(\boldsymbol{\mu})$ depends on the parameter, it can happen that the geometry of a single subdomain $\Omega_{i}(\boldsymbol{\mu})$ depends only on some components of $\boldsymbol{\mu}$, thus the $i$ th local problem depends on $\boldsymbol{\mu}_{i}=\left(\mu_{i_{1}}, \ldots, \mu_{i_{P_{i}}}\right)$, where $\left\{i_{1}, \ldots, i_{P_{i}}\right\} \subseteq\{1, \ldots, P\}$. This fact can be very favourable in terms of the offline computational cost, because the local Greedy algorithm could be performed on a parameter space which has a smaller dimension than the global one.

We discuss two possible definitions of $\mathcal{I}_{i}^{\beta}$, used to construct the local bases.

Method A: approximation of the weak normal derivative of a discrete solution on the fine-grid. First of all, we observe that the weak normal derivative of the fine-grid solution is actually a functional $\mathcal{F}_{h, i}^{\mu} \in\left(V_{h, i}^{\Gamma_{i}}\right)^{\prime}$, which corresponds to an element $w_{h, i}^{\Gamma_{i}}(\boldsymbol{\mu}) \in V_{h, i}^{\Gamma_{i}}$ by the Riesz representation theorem. We then consider a basis $\mathcal{B}^{\Gamma_{i}}=\left\{\phi_{h, 1}^{i}, \ldots, \phi_{N_{\Gamma_{i}}}^{i}\right\}$ of $V_{h, i}^{\Gamma_{i}}$, denote by $\omega_{m}(\boldsymbol{\mu})$ the expansion coefficients of $w_{h, i}^{\Gamma_{i}}(\boldsymbol{\mu})$ with respect to $\mathcal{B}^{\Gamma_{i}}$, and set $n_{B C, i}=N_{\Gamma_{i}}$. Then,

$$
\left\langle\mathcal{F}_{h, i}^{\mu},\left.v_{h, i}\right|_{\Gamma_{i}}\right\rangle=\sum_{m=1}^{n_{B C, i}} \omega_{m}(\boldsymbol{\mu})\left(\phi_{h, m}^{i},\left.v_{h, i}\right|_{\Gamma_{i}}\right)_{\Gamma_{i}} \quad \forall v_{h, i} \in V_{h_{i}} .
$$

Inspired by (2.8), we define the functional $\mathcal{I}_{i}^{\beta}$ (for every $\beta$ ranging from 0 to $n_{B C, i}$, see (2.4)) such that

$$
\left\langle\mathcal{I}_{i}^{\beta},\left.v_{h, i}\right|_{\Gamma_{i}}\right\rangle=\sum_{m=1}^{n_{B C, i}} \mathbb{1}_{\{m\}}(\beta)\left(\phi_{h, m}^{i},\left.v_{h, i}\right|_{\Gamma_{i}}\right)_{\Gamma_{i}} \quad \forall v_{h, i} \in V_{h_{i}}
$$

where $\mathbb{1}_{\{m\}}(\beta)=1$ if and only if $\beta=m$, otherwise it is null (that is $\mathbb{1}_{m}(\beta)=\delta_{m \beta}$ ). In this way, the local Greedy procedure can take into account the Neumann data associated with each interface basis $\phi_{h, i}, i=1, \ldots, N_{\Gamma_{i}}$. 
Method B: Legendre approximation of the normal derivative of the continuous solution. We assume now that the interface $\Gamma_{i}$ of $\Omega_{i}$ is regular (or, at least, it is a finite union of regular components). The idea is now to approximate the normal derivative with a properly chosen $\mathrm{L}^{2}$ orthogonal basis $\left\{\phi_{m}^{i}\right\}_{m=0}^{\infty}$. We write

$$
\frac{\partial u(\boldsymbol{\mu})}{\partial \boldsymbol{n}}=\sum_{m=1}^{\infty} \omega_{m}(\boldsymbol{\mu}) \phi_{m}^{i}
$$

and consider its approximation obtained by truncating the series. We denote the truncated sum with $\widetilde{w}^{\Gamma_{i}}(\boldsymbol{\mu})$ and choose the Legendre polynomials as basis functions. We now define the functional $\mathcal{I}_{i}^{\beta}$ in the following way:

$$
\left\langle\mathcal{I}_{i}^{\beta},\left.v_{h, i}\right|_{\Gamma_{i}}\right\rangle=\sum_{m=1}^{n_{B C, i}} \mathbb{1}_{\{m\}}(\beta)\left(\phi_{m}^{i},\left.v_{h, i}\right|_{\Gamma_{i}}\right)_{\Gamma_{i}} \quad \forall v_{h, i} \in V_{h_{i}} .
$$

We chose $n_{B C, i}$ in order to achieve a good local approximation, as we will discuss later.

\subsection{Online stage}

The elements of $V^{R B}$ are obviously discontinuous functions across subdomain interfaces. To compensate for that, we introduce the following DG-type bilinear form

$$
A_{D G}\left(w^{R B}, v^{R B} ; \boldsymbol{\mu}\right)=\sum_{i=1}^{N_{S}} A_{i}\left(w_{i}^{R B}, v_{i}^{R B} ; \boldsymbol{\mu}\right)+d_{D G}\left(w^{R B}, v^{R B} ; \boldsymbol{\mu}\right)+c_{D G}\left(w^{R B}, v^{R B} ; \boldsymbol{\mu}\right),
$$

where

$$
\begin{aligned}
d_{D G}\left(w^{R B}, v^{R B} ; \boldsymbol{\mu}\right) & =-\int_{\Gamma(\boldsymbol{\mu})} \nu(\boldsymbol{\mu})\left\{\nabla w^{R B}\right\} \cdot \llbracket v^{R B} \rrbracket \mathrm{d} s-\int_{\Gamma(\boldsymbol{\mu})} \nu(\boldsymbol{\mu})\left\{\nabla v^{R B}\right\} \cdot \llbracket w^{R B} \rrbracket \mathrm{d} s \\
c_{D G}\left(w^{R B}, v^{R B} ; \boldsymbol{\mu}\right) & =\frac{\nu(\boldsymbol{\mu}) \gamma}{h} \int_{\Gamma(\boldsymbol{\mu})} \llbracket w^{R B} \rrbracket \cdot \llbracket v^{R B} \rrbracket \mathrm{d} s,
\end{aligned}
$$

for a suitable constant $\gamma>0$. Here $\Gamma(\boldsymbol{\mu})$ is the union of all internal interfaces, and we used the standard notation for jump and average operators, see [7], that on $\bar{\Gamma}_{i j}=\bar{\Omega}_{i} \cap \bar{\Omega}_{j}$ becomes:

$$
\left.\left\{\nabla v^{R B}\right\}\right|_{\Gamma_{i j}(\boldsymbol{\mu})}=\frac{1}{2}\left(\left.\left(\nabla v_{i}^{R B}\right)\right|_{\Gamma_{i j}(\boldsymbol{\mu})}+\left.\left(\nabla v_{j}^{R B}\right)\right|_{\Gamma_{i j}(\boldsymbol{\mu})}\right),\left.\quad \llbracket v^{R B} \rrbracket\right|_{\Gamma_{i j}(\boldsymbol{\mu})}=\left.\left(v_{i}^{R B} \cdot \boldsymbol{n}_{i}\right)\right|_{\Gamma_{i j}(\boldsymbol{\mu})}+\left.\left(v_{j}^{R B} \cdot \boldsymbol{n}_{j}\right)\right|_{\Gamma_{i j}(\boldsymbol{\mu})},
$$

where $\boldsymbol{n}_{k}$ is the normal unit vector pointing outwards $\Omega_{k}(\boldsymbol{\mu}), k=i, j$.

For any given value $\boldsymbol{\mu} \in \mathcal{D}$, the corresponding global reduced approximation takes the following form

$$
\text { find } u^{R B}(\boldsymbol{\mu}) \in V^{R B} \text { such that } A_{D G}\left(u^{R B}(\boldsymbol{\mu}), v^{R B} ; \boldsymbol{\mu}\right)=F\left(v^{R B} ; \boldsymbol{\mu}\right) \quad \forall v^{R B} \in V^{R B},
$$

where $F\left(v^{R B} ; \boldsymbol{\mu}\right)=\sum_{i=1}^{N_{S}} F_{i}\left(v_{i}^{R B} ; \boldsymbol{\mu}\right)$.

\section{Convergence Analysis}

In this section we present the convergence analysis of the DGRBE method, focusing in particular on the approximation properties of the online problem (2.12). We assume that the weak solution $u(\boldsymbol{\mu})$ of the continuous problem (2.1) has $\mathrm{H}^{2}(\Omega)$ regularity. We then set $V=\mathrm{H}_{0}^{1}(\Omega) \cap \mathrm{H}^{2}(\Omega)$ and $V(R B)=V^{R B}+V$. In the following, we will denote with the symbol $\lesssim$ all the inequalities valid up to a multiplicative constant, which can depend also on the parameter $\boldsymbol{\mu}$. We define the norms

$$
\begin{array}{rlrl}
\|v\|_{D G, \boldsymbol{\mu}}^{2} & =\sum_{i=1}^{N_{S}} A_{i}(v, v ; \boldsymbol{\mu})+c_{D G}(v, v ; \boldsymbol{\mu}) & \forall v \in V(R B) & \forall \boldsymbol{\mu} \in \mathcal{D}, \\
\|v\|_{D G, \boldsymbol{\mu}}^{2}=\|v\|_{D G, \boldsymbol{\mu}}^{2}+h^{2} \sum_{i=1}^{N_{S}} \sum_{K \in \mathcal{T}_{h, i}}|v|_{\mathrm{H}^{2}(K)}^{2} & \forall v \in V(R B) & \forall \boldsymbol{\mu} \in \mathcal{D},
\end{array}
$$


and observe that $\left\|v^{R B}\right\|_{D G, \boldsymbol{\mu}}=\left\|v^{R B}\right\|_{D G, \boldsymbol{\mu}}$ for any $v^{R B} \in V^{R B}$, as each element of $V^{R B}$ is piecewise linear. We define also the reference parameter independent norms $\|\cdot\|_{D G}=\|\cdot\|_{D G, \bar{\mu}}$ and $\|\cdot\|_{D G}=\|\cdot\|_{D G, \bar{\mu}}$, which are equivalent to the parameter dependent norms. We observe that, as we are using piecewise linear polynomials, $\left\|v^{R B}\right\|_{D G}=\left\|v^{R B}\right\|_{D G}$ for all $v^{R B} \in V^{R B}$.

We assume than that, for $i=1, \ldots, N_{S}$, the local basis $\left\{\zeta_{1}^{i}, \ldots, \zeta_{N_{i}}^{i}\right\}$ of $V_{i}^{R B}$ satisfies the following orthogonality conditions

$$
\left(\zeta_{j}^{i}, \zeta_{k}^{i}\right)_{V_{i}}=\delta_{j k} j, k=1, \ldots, N_{i}, \quad i=1, \ldots, N_{S},
$$

as guaranteed by the Greedy's algorithm [32,34]. Exploiting (2.7), we observe that we can uniquely express every element of $V^{R B}$ as $v^{R B}=\sum_{i=1}^{N_{S}} v_{i}^{R B}$, and that

$$
\left\|v^{R B}\right\|_{D G}^{2}=\sum_{i=1}^{N_{S}}\left(v_{i}^{R B}, v_{i}^{R B}\right)_{V_{i}}+c_{D G}\left(v^{R B}, v^{R B} ; \overline{\boldsymbol{\mu}}\right),
$$

where the inner product $(\cdot, \cdot)_{V_{i}}$ is defined as in $(2.5)$.

The next proposition states that the form $A_{D G}(\cdot, \cdot ; \boldsymbol{\mu})$ is coercive and continuous with respect to the norms $\|\cdot\|_{D G}$ and $\|\cdot\|_{D G}$, respectively. For the sake of brevity we omit the proof, which is based on standard arguments, cf. $[6,7,38]$.

Proposition 3.1 (Stability of the DGRBE method). The following estimates hold.

(1) For all $\boldsymbol{\mu} \in \mathcal{D}$, there exists $\alpha_{D G}(\boldsymbol{\mu})>0$ such that for all $v^{R B} \in V^{R B}$

$$
A_{D G}\left(v^{R B}, v^{R B} ; \boldsymbol{\mu}\right) \geq \alpha_{D G}(\boldsymbol{\mu})\left\|v^{R B}\right\|_{D G}^{2},
$$

provided the stability parameter $\gamma$ in (2.10) has been chosen large enough.

(2) For all $\boldsymbol{\mu} \in \mathcal{D}$, there exists $K_{D G}(\boldsymbol{\mu})>0$ such that for every $w, v \in V(R B)$

$$
A_{D G}(w, v ; \boldsymbol{\mu}) \leq K_{D G}(\boldsymbol{\mu})\|w\|_{D G}\|v\|_{D G} .
$$

We now prove global error estimates. To ease the notation, we will omit the parameter dependence of the domain. Given a parameter value $\boldsymbol{\mu} \in \mathcal{D}$, the idea is to build $z^{R B}(\boldsymbol{\mu}) \in V^{R B}$ for which it is possible to estimate $\left\|u(\boldsymbol{\mu})-z^{R B}(\boldsymbol{\mu})\right\|_{D G}$. To show that we can bound the approximation error with $\left\|u(\boldsymbol{\mu})-z^{R B}(\boldsymbol{\mu})\right\|_{D G}$, we observe that

$$
\left\|u(\boldsymbol{\mu})-u^{R B}(\boldsymbol{\mu})\right\|_{D G} \leq\left\|u(\boldsymbol{\mu})-z^{R B}(\boldsymbol{\mu})\right\|_{D G}+\left\|z^{R B}(\boldsymbol{\mu})-u^{R B}(\boldsymbol{\mu})\right\|_{D G},
$$

and that, as $A_{D G}\left(u(\boldsymbol{\mu})-u^{R B}(\boldsymbol{\mu}), z^{R B}(\boldsymbol{\mu})-u^{R B}(\boldsymbol{\mu}) ; \boldsymbol{\mu}\right)=0$ by strong consistency,

$$
\begin{aligned}
\left\|z^{R B}(\boldsymbol{\mu})-u^{R B}(\boldsymbol{\mu})\right\|_{D G}^{2} & \leq \frac{1}{\alpha_{D G}(\boldsymbol{\mu})} A_{D G}\left(z^{R B}(\boldsymbol{\mu})-u(\boldsymbol{\mu}), z^{R B}(\boldsymbol{\mu})-u^{R B}(\boldsymbol{\mu}) ; \boldsymbol{\mu}\right) \\
& \leq \frac{K_{D G}(\boldsymbol{\mu})}{\alpha_{D G}(\boldsymbol{\mu})}\left\|z^{R B}(\boldsymbol{\mu})-u(\boldsymbol{\mu})\right\|_{D G}\left\|z^{R B}(\boldsymbol{\mu})-u^{R B}(\boldsymbol{\mu})\right\|_{D G} .
\end{aligned}
$$

We follow the approach used in [7], which first requires a local approximation result. Usually, a piecewise polynomial interpolant is used to provide a local best approximation. As we do not dispose of such an interpolant, we will have to build an element of our local spaces which reasonably provides a good approximation of the continuous solution. We finally observe that, for each $\boldsymbol{\mu} \in \mathcal{D}$, the reduced space built by applying the Greedy's algorithm to the problem (2.5) contains the element

$$
z_{i}^{R B}(\boldsymbol{\mu})=\widetilde{u}_{i}^{R B}(\boldsymbol{\mu}, 0)+w_{i}^{R B}(\boldsymbol{\mu}), \quad \text { where } \quad w_{i}^{R B}(\boldsymbol{\mu})=\sum_{m=1}^{n_{B C, i}} \omega_{m}(\boldsymbol{\mu}) \widetilde{u}_{i}^{R B}(\boldsymbol{\mu}, m),
$$

and the weights $\omega_{m}(\boldsymbol{\mu})$ are defined in (2.8) and (2.9) for Method A and Method B, respectively. Recalling that $\Xi \subset \mathcal{D}$ is the set on which inequality (2.6) rigorously hold, we can state the following results. 
Lemma 3.2 (Method A). Given $\boldsymbol{\mu} \in \Xi$, if the local reduced bases are built using Method $A$, then $z_{i}^{R B}(\boldsymbol{\mu})$ approximates $u_{h, i}(\boldsymbol{\mu}) \in V_{h, i}$, i.e., the restriction to $\Omega_{i}$ of the global fine-grid solution $u_{h}(\boldsymbol{\mu})$ such that

$$
A_{D G}\left(u_{h}(\boldsymbol{\mu}), v_{h} ; \boldsymbol{\mu}\right)=F\left(v_{h} ; \boldsymbol{\mu}\right) \quad \forall v_{h} \in V_{h},
$$

where $V_{h}=\bigoplus_{i=1}^{N_{S}} V_{h, i}$. Moreover it holds that

$$
\begin{aligned}
& \left\|u_{h, i}(\boldsymbol{\mu})-z_{i}^{R B}(\boldsymbol{\mu})\right\|_{\boldsymbol{\mu}, i} \leq \Phi_{\boldsymbol{\mu}}^{A} \varepsilon^{*}, \\
& \left\|u_{h, i}(\boldsymbol{\mu})-z_{i}^{R B}(\boldsymbol{\mu})\right\|_{\mathrm{L}^{2}\left(\Gamma_{i}\right)} \lesssim \Phi_{\boldsymbol{\mu}}^{A} \varepsilon^{*},
\end{aligned}
$$

with $\Phi_{\boldsymbol{\mu}}^{A, i}=\alpha_{i}(\boldsymbol{\mu})^{-1}\left[\left\|\widetilde{F}_{i}\right\|_{V_{h, i}^{\prime}}+N_{\Gamma_{i}} \max _{m}\left(\omega_{m}(\boldsymbol{\mu})\left\|\mathcal{I}_{i}^{m}\right\|_{V_{h, i}^{\prime}}\right)\right]$. Here $N_{\Gamma_{i}}$ is the number of fine-grid interface bases and $V_{h, i}^{\prime}$ is the dual space of $V_{h, i}$.

Proof. We first observe that $u_{h, i}(\boldsymbol{\mu})$ is the solution of

$$
A_{i}\left(u_{h, i}(\boldsymbol{\mu}), v_{h, i} ; \boldsymbol{\mu}\right)=F_{i}\left(v_{h, i} ; \boldsymbol{\mu}\right)+\left\langle\mathcal{F}_{h, i}^{\mu}, v_{h, i}\right\rangle \quad \forall v_{h, i} \in V_{h, i},
$$

where $\mathcal{F}_{h, i}^{\mu} \in\left(V_{h, i}^{\Gamma_{i}}\right)^{\prime}$ can be decomposed as in (2.8). We then note that $u_{h, i}(\boldsymbol{\mu})$ can be written as $u_{h, i}(\boldsymbol{\mu})=$ $\widetilde{u}_{h, i}(\boldsymbol{\mu}, 0)+w_{h, i}(\boldsymbol{\mu})$ where $\widetilde{u}_{h, i}(\boldsymbol{\mu}, 0)$ solves problem $(2.5)$ with $\beta=0$ and $w_{h, i}(\boldsymbol{\mu})=\sum_{m=1}^{N_{\Gamma_{i}}} \omega_{m}(\boldsymbol{\mu}) \widetilde{u}_{h, i}(\boldsymbol{\mu}, m)$. Using (2.6) and observing that

$$
\left\|w_{h, i}(\boldsymbol{\mu})-w_{i}^{R B}(\boldsymbol{\mu})\right\|_{\boldsymbol{\mu}, i} \leq \sum_{m=1}^{N_{\Gamma_{i}}} \omega_{m}(\boldsymbol{\mu})\left\|\widetilde{u}_{h, i}(\boldsymbol{\mu}, m)-\widetilde{u}_{i}^{R B}(\boldsymbol{\mu}, m)\right\|_{\boldsymbol{\mu}, i} \leq \varepsilon^{*} N_{\Gamma_{i}} \max _{m}\left(\omega_{m}(\boldsymbol{\mu})\left\|\mathcal{I}_{i}^{m}\right\|_{V_{h, i}^{\prime}}\right),
$$

estimate (3.4) follows from triangular inequality. As for (3.5), we observe that the norm $\|\cdot\|_{\boldsymbol{\mu}, i}$ is associated with a diffusion reaction operator and, using the standard trace inequality

$$
\left\|v_{i}\right\|_{\mathrm{L}^{2}\left(\Gamma_{i}\right)} \lesssim\left\|v_{i}\right\|_{\mathrm{H}^{1}\left(\Omega_{i}\right)} \quad \forall v_{i} \in \mathrm{H}^{1}\left(\Omega_{i}\right)
$$

cf. [31], we conclude with the desired result.

Lemma 3.3 (Method B). Given $\boldsymbol{\mu} \in \Xi$, if the local reduced bases are built using Method B, then $z_{i}^{R B}(\boldsymbol{\mu})$ is an approximation of $\left.u(\boldsymbol{\mu})\right|_{\Omega_{i}} \in \mathrm{H}_{0}^{1}(\Omega)$, where $u(\boldsymbol{\mu})$ is the weak solution of (2.1). Setting

$$
\mathscr{E}_{i}(\boldsymbol{\mu})=\left\|\frac{\partial u(\boldsymbol{\mu})}{\partial \boldsymbol{n}}-\widetilde{w}^{\Gamma_{i}}(\boldsymbol{\mu})\right\|_{\mathrm{L}^{2}\left(\Gamma_{i}\right)},
$$

where $\widetilde{w}^{\Gamma_{i}}(\boldsymbol{\mu})$ is the truncated sum (2.9), it holds that

$$
\begin{aligned}
& \left\|\left.u(\boldsymbol{\mu})\right|_{\Omega_{i}}-z_{i}^{R B}(\boldsymbol{\mu})\right\|_{\overline{\boldsymbol{\mu}}, i} \lesssim h\left(\|f\|_{\mathrm{L}^{2}\left(\Omega_{i}\right)}+\left\|\frac{\partial u(\boldsymbol{\mu})}{\partial n}\right\|_{\mathrm{L}^{2}\left(\Gamma_{i}\right)}\right)+\mathscr{E}_{i}(\boldsymbol{\mu})+\Phi_{\boldsymbol{\mu}}^{B} \varepsilon^{*} \\
& \left\|\left.u(\boldsymbol{\mu})\right|_{\Omega_{i}}-z_{i}^{R B}(\boldsymbol{\mu})\right\|_{\mathrm{L}^{2}\left(\Gamma_{i}\right)} \lesssim h^{3 / 2}\left(\|f\|_{\mathrm{L}^{2}\left(\Omega_{i}\right)}+\left\|\frac{\partial u(\boldsymbol{\mu})}{\partial n}\right\|_{\mathrm{L}^{2}\left(\Gamma_{i}\right)}\right)+\mathscr{E}_{i}(\boldsymbol{\mu})+\Phi_{\boldsymbol{\mu}}^{B} \varepsilon^{*},
\end{aligned}
$$

with $\Phi_{\boldsymbol{\mu}}^{B, i}=\alpha_{i}(\boldsymbol{\mu})^{-1}\left[\left\|\widetilde{F}_{i}\right\|_{V_{h, i}^{\prime}}+\sum_{m=1}^{M_{B C}} \omega_{m}(\boldsymbol{\mu})\left\|\mathcal{I}_{i}^{m}\right\|_{V_{h, i}^{\prime}}\right]$. Here $M_{B C}$ is the number of Legendre polynomials considered on each interface and $V_{h, i}^{\prime}$ is the dual space of $V_{h, i}$. 
Proof. We first split the restriction of the exact solution to $\Omega_{i}$ as $\left.u(\boldsymbol{\mu})\right|_{\Omega_{i}}=\stackrel{\varkappa}{i}_{i}(\boldsymbol{\mu})+w_{i}(\boldsymbol{\mu})$, where $\stackrel{\cup}{i}_{i}(\boldsymbol{\mu})$ and $w_{i}(\boldsymbol{\mu})$ are such that

$$
A_{i}\left(\stackrel{\circ}{i}_{i}(\boldsymbol{\mu}), v_{i} ; \boldsymbol{\mu}\right)=F_{i}\left(v_{i} ; \boldsymbol{\mu}\right), \quad A_{i}\left(w_{i}(\boldsymbol{\mu}), v_{i} ; \boldsymbol{\mu}\right)=\left(\nu(\boldsymbol{\mu}) \frac{\partial u(\boldsymbol{\mu})}{\partial \boldsymbol{n}},\left.v_{i}\right|_{\Gamma_{i}}\right)_{\Gamma_{i}},
$$

for all $v_{i} \in V_{i}$. We then define $\widetilde{w}_{i}(\boldsymbol{\mu})$ such that

$$
A_{i}\left(\widetilde{w}_{i}(\boldsymbol{\mu}), v_{i} ; \boldsymbol{\mu}\right)=\left(\widetilde{w}^{\Gamma_{i}}(\boldsymbol{\mu}),\left.v_{i}\right|_{\Gamma_{i}}\right)_{\Gamma_{i}} \quad \forall v_{i} \in V_{i},
$$

where $\widetilde{w}^{\Gamma_{i}}(\boldsymbol{\mu})$ is the polynomial expansion (2.9) truncated after the first $M_{B C}$ terms. Moreover, it holds that $\widetilde{w}_{i}(\boldsymbol{\mu})=\sum_{N=1}^{M_{B C}} \omega_{m}(\boldsymbol{\mu}) \psi_{m, i}(\boldsymbol{\mu})$, where $\psi_{m, i}(\boldsymbol{\mu}) \in V_{i}, m=1, \ldots, M$, are the harmonic extensions of the basis of Neumann boundary data, i.e.,

$$
A_{i}\left(\psi_{m, i}(\boldsymbol{\mu}), v_{i} ; \boldsymbol{\mu}\right)=\left(\phi_{m}(\boldsymbol{\mu}),\left.v_{i}\right|_{\Gamma_{i}}\right)_{\Gamma_{i}} \quad \forall v_{i} \in V_{i} .
$$

Note that $\widetilde{u}_{h, i}(\boldsymbol{\mu}, m)$ is the FE approximation of $\psi_{m, i}(\boldsymbol{\mu})$, then the FE approximation of $\widetilde{w}_{i}(\boldsymbol{\mu})$ is $\widetilde{w}_{h, i}(\boldsymbol{\mu})=$ $\sum_{m=1}^{M_{B C}} \omega_{m}(\boldsymbol{\mu}) \widetilde{u}_{h, i}(\boldsymbol{\mu}, m)$. By triangular inequality,

$$
\begin{aligned}
\left\|\left.u(\boldsymbol{\mu})\right|_{\Omega_{i}}-z_{i}^{R B}(\boldsymbol{\mu})\right\|_{\overline{\boldsymbol{\mu}}, i} \leq & \left\|\mathrm{i}_{i}(\boldsymbol{\mu})-\widetilde{u}_{h, i}(\boldsymbol{\mu}, 0)\right\|_{\overline{\boldsymbol{\mu}}, i}+\left\|\widetilde{u}_{h, i}(\boldsymbol{\mu}, 0)-\widetilde{u}_{i}^{R B}(\boldsymbol{\mu}, 0)\right\|_{\overline{\boldsymbol{\mu}}, i} \\
& +\left\|w_{i}(\boldsymbol{\mu})-\widetilde{w}_{i}(\boldsymbol{\mu})\right\|_{\overline{\boldsymbol{\mu}}, i}+\left\|\widetilde{w}_{i}(\boldsymbol{\mu})-\widetilde{w}_{h, i}(\boldsymbol{\mu})\right\|_{\bar{\mu}, i}+\left\|\widetilde{w}_{h, i}(\boldsymbol{\mu})-w_{R B, i}(\boldsymbol{\mu})\right\|_{\overline{\boldsymbol{\mu}}, i} .
\end{aligned}
$$

Recalling the standard error estimates of the FE method (cf. [31]), exploiting (2.6) and reasoning as in (3.6) inequality (3.8) follows. To prove (3.9), we need the following trace inequalities:

$$
\|v\|_{\mathrm{L}^{2}(e)}^{2} \lesssim h_{e}^{-1}\|v\|_{\mathrm{L}^{2}(K)}^{2}+h_{e}|v|_{\mathrm{H}^{1}(K)}^{2} \quad \forall v \in \mathrm{H}^{1}(K) \quad \forall K \in \mathcal{T}_{h},
$$

cf. [7], where $e$ is an edge of $K$, that implies

$$
\left\|v_{i}\right\|_{\mathrm{L}^{2}\left(\Gamma_{i}\right)}^{2} \lesssim h^{-1}\left\|v_{i}\right\|_{\mathrm{L}^{2}\left(\Omega_{i}\right)}^{2}+h\left|v_{i}\right|_{\mathrm{H}^{1}\left(\Omega_{i}\right)}^{2} \quad \forall v_{i} \in \mathrm{H}^{1}\left(\Omega_{i}\right), \quad i=1, \ldots, N_{S} .
$$

We then decompose the $\left\|\left.u(\boldsymbol{\mu})\right|_{\Omega_{i}}-z_{i}^{R B}(\boldsymbol{\mu})\right\|_{\mathrm{L}^{2}\left(\Gamma_{i}\right)}$ as done in (3.10). By (3.11) and the standard FE error estimates, we can bound the FE parts as follows

$$
\left\|\stackrel{i}{i}_{i}(\boldsymbol{\mu})-\widetilde{u}_{h, i}(\boldsymbol{\mu}, 0)\right\|_{\mathrm{L}^{2}\left(\Gamma_{i}\right)}+\left\|\widetilde{w}_{i}(\boldsymbol{\mu})-\widetilde{w}_{h, i}(\boldsymbol{\mu})\right\|_{\mathrm{L}^{2}\left(\Gamma_{i}\right)} \lesssim h^{3 / 2}\left(\|f\|_{\mathrm{L}^{2}\left(\Omega_{i}\right)}+\left\|\frac{\partial u(\boldsymbol{\mu})}{\partial n}\right\|_{\mathrm{L}^{2}\left(\Gamma_{i}\right)}\right) .
$$

The thesis follows bounding the remaining parts using the trace inequality (3.7).

In order to prove a global approximation estimate, we use an argument similar to that used in ([7], Sect. 4.3). We also exploit the following inequality, whose proof is shown in Appendix C,

$$
\frac{1}{h} \sum_{\left\{i, j: \bar{\Omega}_{i} \cap \bar{\Omega}_{j} \neq \emptyset\right\}}\left\|\llbracket v_{h} \rrbracket\right\|_{\mathrm{L}^{2}\left(\Gamma_{i j}\right)}^{2} \lesssim \frac{1}{h} \sum_{i=1}^{N_{S}}\left\|v_{h, i}\right\|_{\mathrm{H}^{1}\left(\Omega_{i}\right)}^{2} \quad \forall v_{h} \in V_{h} .
$$

We define: $z^{R B}(\boldsymbol{\mu})=\sum_{i=1}^{N_{S}} z_{i}^{R B}(\boldsymbol{\mu})$. The following result holds.

Theorem 3.4. Let $\boldsymbol{\mu} \in \Xi$. Let $u(\boldsymbol{\mu})$ be the exact weak solution of problem (2.1) and let $u_{A}^{R B}(\boldsymbol{\mu})$ and $u_{B}^{R B}(\boldsymbol{\mu})$ be the approximated solutions obtained with Method $A$ and Method B, respectively. Then,

$$
\begin{aligned}
&\left\|u(\boldsymbol{\mu})-u_{A}^{R B}(\boldsymbol{\mu})\right\|_{D G} \lesssim h\|f\|_{\mathrm{L}^{2}\left(\Omega_{i}\right)}+\sqrt{N_{S}}\left(1+\frac{1}{\sqrt{h}}\right) \Phi_{\boldsymbol{\mu}}^{A} \varepsilon^{*}, \\
&\left\|u(\boldsymbol{\mu})-u_{B}^{R B}(\boldsymbol{\mu})\right\|_{D G} \lesssim h\|f\|_{\mathrm{L}^{2}(\Omega)}+\left(1+\frac{1}{\sqrt{h}}\right) \sum_{i=1}^{N_{S}} \mathscr{E}_{i}(\boldsymbol{\mu})+\sqrt{N_{S}}\left(1+\frac{1}{\sqrt{h}}\right) \Phi_{\boldsymbol{\mu}}^{B} \varepsilon^{*},
\end{aligned}
$$


where $\Phi_{\boldsymbol{\mu}}^{A}=\max _{i} \Phi_{\boldsymbol{\mu}}^{A, i}$ and $\Phi_{\boldsymbol{\mu}}^{B}=\max _{i} \Phi_{\boldsymbol{\mu}}^{B, i}$. Here $\Phi_{\boldsymbol{\mu}}^{A, i}, \Phi_{\boldsymbol{\mu}}^{B, i}$ and $\mathscr{E}_{i}(\boldsymbol{\mu})$ are defined as in Lemmas 3.2 and 3.3 . All the hidden constants depend on the domain $\Omega$ and the parameter $\boldsymbol{\mu}$, but are independent of $h$ and $\varepsilon^{*}$.

Proof. Let us start with estimate (3.13). First of all we observe that the solution $u_{h}(\boldsymbol{\mu})$ of $(3.3)$ is such that

$$
\left\|u(\boldsymbol{\mu})-u_{h}(\boldsymbol{\mu})\right\|_{D G} \lesssim h\|f\|_{\mathrm{L}^{2}(\Omega)},
$$

see, for instance, [35]. We then observe that as $V_{h}$ and thus $V^{R B}$ are piecewise polynomials spaces, it is sufficient to estimate the error $\left\|u_{h}(\boldsymbol{\mu})-z^{R B}(\boldsymbol{\mu})\right\|_{D G}$. More precisely, we observe that we need just to estimate the jump terms, as the others are already bounded by (3.4). Using inequality (3.12) together with Lemma 3.2, we obtain

$$
\frac{\gamma}{h} \sum_{\left\{i, j: \bar{\Omega}_{i} \cap \bar{\Omega}_{j} \neq \emptyset\right\}}\left\|\llbracket u_{h}(\boldsymbol{\mu})-z^{R B}(\boldsymbol{\mu}) \rrbracket\right\|_{L^{2}\left(\Gamma_{i j}\right)}^{2} \lesssim \frac{1}{h} \sum_{i=1}^{N_{S}}\left\|u_{h, i}(\boldsymbol{\mu})-z_{i}^{R B}(\boldsymbol{\mu})\right\|_{\overline{\boldsymbol{\mu}}, i}^{2} \lesssim\left(\sqrt{\frac{N_{S}}{h}} \Phi_{\boldsymbol{\mu}}^{A} \varepsilon^{*}\right)^{2}
$$

As for (3.14), it is sufficient to find an upper bound for the jump term of $\|\cdot\|_{D G}$, because the other terms can be controlled by the local estimate (3.8) and by observing that

$$
\sum_{i=1}^{N_{S}} \sum_{K \in \mathcal{T}_{h, i}}\left|u(\boldsymbol{\mu})-z^{R B}(\boldsymbol{\mu})\right|_{\mathrm{H}^{2}(K)}^{2}=|u(\boldsymbol{\mu})|_{\mathrm{H}^{2}(\Omega)}^{2} \lesssim\|f(\boldsymbol{\mu})\|_{\mathrm{L}^{2}(\Omega)}^{2} .
$$

Thanks to inequality (3.7), reasoning as in (3.15) and recalling Lemma 3.3, we have

$$
\frac{\gamma}{h} \sum_{\left\{i, j: \bar{\Omega}_{i} \cap \bar{\Omega}_{j} \neq \emptyset\right\}}\left\|\llbracket u(\boldsymbol{\mu})-z^{R B}(\boldsymbol{\mu}) \rrbracket\right\|_{L^{2}\left(\Gamma_{i j}\right)}^{2} \lesssim\left(h\|f\|_{L^{2}(\Omega)}+\frac{1}{\sqrt{h}} \sum_{i=1}^{N_{S}} \mathscr{E}_{i}(\boldsymbol{\mu})+\sqrt{\frac{N_{S}}{h}} \Phi_{\boldsymbol{\mu}}^{B} \varepsilon^{*}\right)^{2},
$$

where we have also used that

$$
\sum_{i=1}^{N_{S}}\left\|\frac{\partial u(\boldsymbol{\mu})}{\partial n}\right\|_{\mathrm{L}^{2}\left(\Gamma_{i}\right)} \lesssim \sum_{i=1}^{N_{S}}\|\nabla u(\boldsymbol{\mu})\|_{\mathrm{L}^{2}\left(\Omega_{i}\right)} \lesssim\|\nabla u(\boldsymbol{\mu})\|_{\mathrm{L}^{2}(\Omega)} \lesssim\|f\|_{\mathrm{L}^{2}(\Omega)},
$$

and the thesis follows.

Remark 3.5. Note that for both Methods A and B the contribution to the error due to the local RB increases as the square root of the number of subdomains. This is actually reasonable because, by (2.6), we can control only the RB approximation error committed on a single subdomain. Then, since the global absolute error depends on the square root of the sum of the squared local errors, the dependence on $\sqrt{N_{S}}$ is expectable.

Remark 3.6. We observe that the constant $\Phi_{\boldsymbol{\mu}}^{A, i}=\alpha_{i}(\boldsymbol{\mu})^{-1}\left[\left\|\widetilde{F}_{i}\right\|_{V_{h, i}^{\prime}}+N_{\Gamma_{i}} \max _{m}\left(\omega_{m}(\boldsymbol{\mu})\left\|\mathcal{I}_{i}^{m}\right\|_{V_{h, i}^{\prime}}\right)\right]$, cf. Lemma 3.2, may a priori depend on $h$. Indeed, for each subdomain $\Omega_{i}$ and for each FE basis function on $\Gamma_{i}$ we have $\left\|\mathcal{I}_{i}^{m}\right\|_{V_{h, i}^{\prime}} \lesssim\left\|\phi_{h, m}^{i}\right\|_{L^{2}\left(\Gamma_{i}\right)} \lesssim h^{1 / 2}$. Therefore, since $N_{\Gamma} \approx h^{-1}$, we have that $\Phi_{\mu}^{A, i} \lesssim h^{-1 / 2}$. Nevertheless, our numerical computations reveal that this bound might not be sharp, as $\Phi_{\boldsymbol{\mu}}^{A, i}$ is very likely to be independent of $h$. More precisely, in Figure 4 we will display that $\max _{m}\left\|\mathcal{I}_{i}^{m}\right\|_{V_{h, i}^{\prime}}$ is of order $h$ and in such a case $\Phi_{\mu}^{A, i}$ is independent of the mesh size. A theoretical justification of this behaviour is under investigation.

Remark 3.7. The quality of the global approximation given by Method B depends on how well the normal derivative of $u(\boldsymbol{\mu})$ on the interfaces can be approximated by a polynomial expansion, on each internal interface. In our numerical tests, shown in Section 5 , we chose $M_{B C} \approx h^{-1 / 2}$. This choice can be motivated employing the approximation properties in average of the Legendre polynomials, provided that we assume high regularity 
on the solution $u(\boldsymbol{\mu})$. More precisely, if $u(\boldsymbol{\mu}) \in \mathrm{H}^{5}(\Omega)$ then $\frac{\partial u(\boldsymbol{\mu})}{\partial \boldsymbol{n}}$ has (at least) $\mathrm{H}^{3}$ regularity on each regular component of the internal interface. Thus, the following approximation result holds:

$$
\left\|\frac{\partial u(\boldsymbol{\mu})}{\partial \boldsymbol{n}}-\widetilde{w}^{\Gamma_{i}}(\boldsymbol{\mu})\right\|_{\mathrm{L}^{2}\left(\Gamma_{i}\right)} \lesssim M^{-3}\left|\frac{\partial u(\boldsymbol{\mu})}{\partial \boldsymbol{n}}\right|_{\mathrm{H}^{3}\left(\Gamma_{i}\right)},
$$

$c f$. [12]. In this way, the error due to the approximation of the normal derivative on the interfaces scales as $h$. A possible alternative to the requirement of a high regularity of the solution is the introduction of a suitable a posteriori error estimator which allow to automatically tune the number of polynomial bases at the interface. The latter approach is currently under investigation [5]. It is worth to be noted that, even if we consider $M_{B C} \approx h^{-1 / 2}$, the constant $\Phi_{\mu}^{B}$ is independent of $h$. To show this it is sufficient to consider the decay of the coefficients $\omega_{m}(\boldsymbol{\mu})$ of the Legendre expansion of the normal derivative $\left.\frac{\partial u(\boldsymbol{\mu})}{\partial \boldsymbol{n}}\right|_{\Gamma_{i}}$. To this end, we exploit the following proposition.

Proposition 3.8. Let $I=(-1,1), f \in \mathrm{H}^{k}(I), k \geq 2$, and let $P_{n}$ be the $n$th Legendre polynomial, $n \in \mathbb{N}$. Then

$$
f=\sum_{n=0}^{\infty} a_{n} P_{n}, \quad \text { with } \quad a_{n}=\left(n+\frac{1}{2}\right) \int_{I} f P_{n}
$$

and

$$
\left|a_{n}\right| \lesssim \frac{1}{n^{k-1}}\left\|f^{(k)}\right\|_{\mathrm{L}^{2}(I)}
$$

The proof of Proposition 3.8 is based on the properties of Legendre polynomials and follows the same lines of ([37], Thm. 2.1); for this reason it has been omitted. Thanks to Proposition 3.8 and our regularity assumption on $u(\boldsymbol{\mu})$ we have that $\sum_{m=1}^{M_{B C}} \omega_{m}(\boldsymbol{\mu}) \leq C<\infty$, thus $\Phi_{\boldsymbol{\mu}}^{B}$ is independent of $h$.

\subsection{Spectral bounds}

We prove now some spectral bounds on the condition number of the matrix associated with the online problem (2.12) through the basis $\mathcal{B}_{R B}$. We observe that for $i=1, \ldots, N_{S}$, every element $v_{i}^{R B} \in V_{i}^{R B}$ can be expressed as $v_{i}^{R B}=\sum_{j=1}^{N_{i}} \mathrm{v}_{j}^{i} \zeta_{j}^{i}$. We set $\mathbf{v}_{i}=\left(\mathrm{v}_{1}^{i}, \ldots, \mathrm{v}_{N_{i}}^{i}\right)$. Then, each $v^{R B} \in V^{R B}$ is associated with a vector $\mathbf{v}^{R B}=\left(\mathbf{v}_{1}, \ldots, \mathbf{v}_{N} s\right)$.

Lemma 3.9. It holds:

$$
\left\|v_{i}^{R B}\right\|_{V_{i}}^{2}=\left|\mathbf{v}_{i}\right|^{2} \quad \forall v_{i}^{R B} \in V_{i}^{R B}, i=1, \ldots, N_{S},
$$

where $|\cdot|$ denotes the Euclidean norm.

Proof. Thanks to the orthogonality assumption (3.1), for $i=1, \ldots, N_{S}$, it holds

$$
\left\|v_{i}^{R B}\right\|_{V_{i}}^{2}=\left(v_{i}^{R B}, v_{i}^{R B}\right)_{V_{i}}=\left(\sum_{j=1}^{N_{i}} \mathrm{v}_{j}^{i} \zeta_{j}^{i}, \sum_{k=1}^{N_{i}} \mathrm{v}_{k}^{i} \zeta_{k}^{i}\right)_{V_{i}}=\sum_{j, k=1}^{N_{i}} \mathrm{v}_{j}^{i} \mathrm{v}_{k}^{i}\left(\zeta_{j}^{i}, \zeta_{k}^{i}\right)_{V_{i}}=\mathbf{v}_{i}^{\mathrm{T}} \mathbf{v}_{i} .
$$

Lemma 3.10. It holds

$$
c_{D G}\left(v^{R B}, v^{R B} ; \overline{\boldsymbol{\mu}}\right) \lesssim \frac{\nu(\overline{\boldsymbol{\mu}}) \gamma}{h}\left|\mathbf{v}^{R B}\right|^{2} \quad \forall v^{R B} \in V^{R B},
$$

where $\gamma$ is the penalization coefficient defined in (2.11) and the hidden constant depends only on the reference domain $\Omega$. 
Proof. Let us consider the interface $\Gamma_{i j}$ between $\Omega_{i}$ and $\Omega_{j}$. Recalling that:

$$
\left\|v_{k}\right\|_{\mathrm{L}^{2}\left(\Gamma_{i j}\right)} \leq C_{\Gamma_{i j}}\left\|v_{k}\right\|_{V_{k}} \forall v_{k} \in V_{k}, k=i, j,
$$

cf. [10], we observe that, using the Schwarz's inequality and (3.17)

$$
\left|\int_{\Gamma_{i j}} \llbracket v^{R B} \rrbracket^{2}\right| \leq C_{\Gamma_{i j}}^{2}\left(\left\|v_{i}^{R B}\right\|_{V_{i}}^{2}+\left\|v_{j}^{R B}\right\|_{V_{j}}^{2}+2\left\|v_{i}^{R B}\right\|_{V_{i}}\left\|v_{j}^{R B}\right\|_{V_{j}}\right) \lesssim C_{\Gamma_{i j}}^{2}\left(\left\|v_{i}^{R B}\right\|_{V_{i}}^{2}+\left\|v_{j}^{R B}\right\|_{V_{j}}^{2}\right) .
$$

Using Lemma 3.9 we finally have

$$
\left|\int_{\Gamma_{i j}} \llbracket v^{R B} \rrbracket^{2}\right| \lesssim C_{\Gamma_{i j}}^{2}\left(\left|\mathbf{v}_{i}\right|^{2}+\left|\mathbf{v}_{j}\right|^{2}\right)
$$

Summing (3.18) over $i$ and $j$, we get

$$
\left|\int_{\Gamma} \llbracket v^{R B} \rrbracket^{2}\right| \lesssim C_{\Gamma_{i j}}^{2}\left|\mathbf{v}_{R B}\right|^{2}
$$

where the hidden constant depends on the maximum number of neighbouring subdomains a given subdomain can have. Recalling (2.11), the thesis follows.

From Lemmas 3.9 and 3.10 we can obtain the following result.

Theorem 3.11. The minimum and maximum eigenvalues of $\mathbf{A}_{R B}$ satisfy

$$
\lambda_{\min }\left(\mathbf{A}_{R B}\right) \geq \alpha_{D G}(\boldsymbol{\mu}), \quad \lambda_{\max }\left(\mathbf{A}_{R B}\right) \lesssim K_{D G}(\boldsymbol{\mu})\left(1+\frac{\nu(\overline{\boldsymbol{\mu}}) \gamma}{h}\right),
$$

for every $\boldsymbol{\mu} \in \mathcal{D}$, where $\gamma$ is defined in (2.11). The condition number of $\mathbf{A}_{R B}$ can therefore be bounded by

$$
\kappa\left(\mathbf{A}_{R B}\right) \lesssim \frac{K_{D G}(\boldsymbol{\mu})}{\alpha_{D G}(\boldsymbol{\mu})}\left(1+\frac{\nu(\overline{\boldsymbol{\mu}}) \gamma}{h}\right) .
$$

\section{Preconditioning the Online System}

In this section we propose a possible two-level preconditioner to efficiently solve the online system. More precisely, we aim to find a preconditioner for the parameter independent bilinear form

$$
B\left(w_{h}, v_{h}\right)=\sum_{i=1}^{N_{S}}\left(w_{h, i}, v_{h, i}\right)_{V_{i}}+c_{D G}\left(v_{h}, w_{h} ; \overline{\boldsymbol{\mu}}\right),
$$

which is the scalar product associated with the norm $\|\cdot\|_{D G}$ (see (3.2)) and is spectrally equivalent to the form $A_{D G}(\cdot, \cdot ; \boldsymbol{\mu})$ (see Prop. 3.1). In the following, $\mathbf{B}$ will be the matrix associated with $B$ through the basis $\mathcal{B}_{R B}$ and $\mathbf{P}$ the preconditioner of the online algebraic system.

The key ingredient of our two-level preconditioner is the construction of a coarse solver. For $i=1, \ldots, N_{S}$ we define a parameter independent coarse triangulation $\mathcal{T}_{H, i}$, and assume that each fine triangulation $\mathcal{T}_{h, i}$ introduced in Section 2.1 is a refinement of $\mathcal{T}_{H, i}$. On each subdomain we define the local reduced space $V_{i}^{R B}$ as

$$
V_{i}^{R B}=V_{H, i} \oplus V_{N_{i}, i},
$$

where $V_{H, i}$ is the piecewise discontinuous linear FE space associated with $\mathcal{T}_{H, i}$, while $V_{N_{i}, i}$ is the space spanned by local reduced basis functions computed in $V_{h, i}$. We denote with $\mathcal{B}_{H, i}$ the standard FE basis of $V_{H, i}$ and 
with $\mathcal{B}_{N_{i}, i}$ the basis of $V_{N_{i}, i}$. A basis for $V_{i}^{R B}$ is then $\mathcal{B}_{R B, i}=\mathcal{B}_{H, i} \cup \mathcal{B}_{N_{i}, i}$. We show in Appendix B how to build a basis which satisfies the direct sum assumption in (4.1). The offline-online decomposition of the method enriched with a coarse space is very similar to that shown in Section 2. The main difference is that now the global reduced space is defined as

$$
V^{R B}=\bigoplus_{i=1}^{N_{S}} V_{i}^{R B}=\bigoplus_{i=1}^{N_{S}} V_{H, i} \oplus \bigoplus_{i=1}^{N_{S}} V_{N_{i}, i}
$$

We note that $V_{H}=\bigoplus_{i=1}^{N_{S}} V_{H, i}$ is a non-conforming global coarse approximation space.

Remark 4.1. As regards the stability of the online problem associated with the reduced space (4.2) and the approximation properties, the results proven in Section 3 still hold.

\subsection{Two level Schwarz preconditioner}

In this section we introduce a two level Schwarz preconditioner based on the reduced space enriched with a coarse FE space, following the approach used in [2-4]. We recall that the global space is the DGRBE space $V^{R B}$ defined in (4.2), we then define the global coarse space $W_{0}=\bigoplus_{i=1}^{N_{S}} V_{H, i}$, and the local spaces $W_{i}=V_{i}^{R B}$, for $i=1, \ldots, N_{S}$ (see (4.1) for the definition). We observe that $W_{0} \subseteq \bigoplus_{i=1}^{N_{S}} W_{i}=V^{R B}$.

Let $R_{i}^{\mathrm{T}}: W_{i} \rightarrow V^{R B}$ be the inclusion $W_{i} \rightarrow V^{R B}$. Now, it is possible to define the local operators $B_{i}$, $i=0, \ldots, N_{S}$ such that

$$
B_{i}\left(w_{i}, v_{i}\right)=B\left(R_{i}^{\mathrm{T}} w_{i}, R_{i}^{\mathrm{T}} v_{i}\right) \quad \forall w_{i}, v_{i} \in W_{i} .
$$

We then introduce some projection-like operators $P_{i}=R_{i}^{\mathrm{T}} \widetilde{P}_{i}: V^{R B} \rightarrow R_{i}^{\mathrm{T}} W_{i}$, for $i=0, \ldots, N_{S}$, where $\widetilde{P}_{i}: V^{R B} \rightarrow W_{i}$ is such that:

$$
B_{i}\left(\widetilde{P}_{i} w, v_{i}\right)=B\left(w, R_{i}^{\mathrm{T}} v_{i}\right) \quad \forall v_{i} \in W_{i} .
$$

The two level additive Schwarz preconditioner is then defined by $P_{a d}=\sum_{i=0}^{N_{S}} P_{i}$. Employing the matrix notation we have $\mathbf{P}_{a d}=\mathbf{P}^{-1} \mathbf{B}$ with $\mathbf{P}^{-1}=\sum_{i=0}^{N_{S}} \mathbf{R}_{i}^{\mathrm{T}} \mathbf{B}_{i}^{-1} \mathbf{R}_{i}$, being $\mathbf{R}_{i}^{\mathrm{T}}$ and $\mathbf{B}_{i}$ the matrix representation of $R_{i}^{\mathrm{T}}$ and $B_{i}(\cdot, \cdot)$, respectively.

We next provide an estimate for the condition number of the preconditioned matrix $\mathbf{P}^{-1} \mathbf{B}$. The arguments used are similar to [2]. Given $w \in V^{R B}$, we define $w_{0} \in W_{0}$ such that:

$$
w_{0}=\sum_{i=1}^{N_{S}} w_{0}^{i}, \quad w_{0}^{i}=\left.\Pi_{\mathrm{L}^{2}}^{V_{H, i}} w\right|_{\Omega_{i}}, \quad i=1, \ldots, N_{S}
$$

where $\Pi_{\mathrm{L}^{2}}^{V_{H, i}}$ is the $\mathrm{L}^{2}$ projection onto $V_{H, i}$. It holds that:

$$
\left\|w-w_{0}^{i}\right\|_{\mathrm{L}^{2}\left(\Omega_{i}\right)} \lesssim H|w|_{\mathrm{H}^{1}\left(\Omega_{i}\right)}, \quad\left|w_{0}^{i}\right| \lesssim|w|_{\mathrm{H}^{1}\left(\Omega_{i}\right)},
$$

for $i=1, \ldots, N_{S}, c f$. [9]. We now report some preliminary lemmas whose proofs are based upon standard arguments ( $c f .[2])$.

Lemma 4.2. For any $w, v \in V^{R B}$, we consider their unique decompositions as $w=\sum_{i=1}^{N_{S}} R_{i}^{\mathrm{T}} w_{i}, v=$ $\sum_{i=1}^{N_{S}} R_{i}^{\mathrm{T}} v_{i}$, with $w_{i}, v_{i} \in W_{i}$ for $i=1, \ldots, N_{S}$. It holds that:

$$
B(w, v)=\sum_{i=1}^{N_{S}} B_{i}\left(w_{i}, w_{i}\right)+I(w, v), \quad \text { where } I(w, v)=\frac{\nu(\overline{\boldsymbol{\mu}})}{h} \sum_{\substack{i, j=1 \\ i<j}}^{N_{S}} \int_{\Gamma_{i j}} u_{i} \boldsymbol{n}_{i} \cdot v_{j} \boldsymbol{n}_{j}+u_{j} \boldsymbol{n}_{j} \cdot v_{i} \boldsymbol{n}_{i} \mathrm{~d} s .
$$


Moreover,

$$
|I(w, w)| \lesssim \gamma \frac{1}{H h}\|w\|_{\mathrm{L}^{2}(\Omega)}^{2}+\gamma \frac{H}{h} \sum_{i=1}^{N_{S}}|w|_{\mathrm{H}^{1}\left(\Omega_{i}\right)}^{2},
$$

where the hidden constant is independent of the mesh sizes $h$ and $H$ and of the penalty parameter $\gamma$.

Lemma 4.3. For any $w \in V^{R B}$, let $w_{0}$ be the piecewise $\mathrm{L}^{2}$ projection defined in (4.3). Then the following estimates hold:

$$
B_{0}\left(w_{0}, w_{0}\right) \lesssim\left(2+\gamma \frac{H}{h}\right) B(w, w), \quad B\left(w-\widetilde{w}_{0}, w-\widetilde{w}_{0}\right) \lesssim\left(1+\gamma \frac{H}{h}\right) B(w, w)
$$

where the hidden constant is independent of $h, H$ and $\gamma$.

Lemma 4.4 (Stable decomposition). For any $w \in V^{R B}$, let $w_{0}$ be the element of $W_{0}$ defined by (4.3) and let $w_{i} \in W_{i}, i=1, \ldots, N_{S}$, be the uniquely determined elements such that $w-R_{0}^{\mathrm{T}} w_{0}=\sum_{i=1}^{N_{S}} R_{i}^{\mathrm{T}} w_{i}$. Then:

$$
\sum_{i=0}^{N_{S}} B_{i}\left(w_{i}, w_{i}\right) \leq \gamma C_{0}^{2} B(w, w), \quad \text { with } \quad C_{0}^{2}=O\left(\frac{H}{h}\right)
$$

Proof. We denote by $\widetilde{w}_{0}=R_{0}^{\mathrm{T}} w_{0}$. We have that

$$
\sum_{i=0}^{N_{S}} B_{i}\left(w_{i}, w_{i}\right)=B\left(w-\widetilde{w}_{0}, w-\widetilde{w}_{0}\right)+B_{0}\left(w_{0}, w_{0}\right)-I\left(w-\widetilde{w}_{0}, w-\widetilde{w}_{0}\right) .
$$

We observe that, thanks to Lemma 4.2, and to (4.4), we have

$$
\left|I\left(w-\widetilde{w}_{0}, w-\widetilde{w}_{0}\right)\right| \lesssim \gamma \frac{1}{H h}\left\|w-\widetilde{w}_{0}\right\|_{\mathrm{L}^{2}(\Omega)}+\gamma \frac{H}{h} \sum_{i=1}^{N_{S}}\left|w-\widetilde{w}_{0}\right|_{\mathrm{H}^{1}\left(\Omega_{i}\right)}^{2} \lesssim \gamma \frac{H}{h} \sum_{i=1}^{N_{S}}|w|_{\mathrm{H}^{1}\left(\Omega_{i}\right)}^{2} \lesssim \gamma \frac{H}{h} B(w, w) .
$$

Exploiting Lemma 4.3 we can conclude.

We can finally prove the following proposition about the condition number of the preconditioned ma$\operatorname{trix} \mathbf{P}^{-1} \mathbf{B}$.

Proposition 4.5. The following estimate holds

$$
\kappa\left(\mathbf{P}^{-1} \mathbf{B}\right) \leq \gamma C_{0}^{2}(2+M) \lesssim \gamma(2+M) \frac{H}{h},
$$

where $M$ is the maximum neighbours of each subdomain.

Proof. The proof is similar to [2], Theorem 5.1, and follows the general theory of Schwarz's methods, see [36].

\section{Numerical RESUlts}

In this section we show some numerical tests in order to validate the theoretical results presented in the previous sections. We recall that we denote with "Method A" and "Method B" the strategies for the construction of the local basis introduced in Section 2.1. In all the following examples, the tolerance of the Greedy's algorithm has been set equal to $\varepsilon^{*}=10^{-5}$ and the coefficient $\gamma$ appearing in the penalization term $c_{D G}$ defined in $(2.11)$ has been chosen as $\gamma=10$. The computations have been performed in MATLAB ${ }^{\circledR}$; the local offline stages have been carried out employing the rbMIT $^{\complement}$ library [21]. 
TABle 1. Example 1. Dimensions of the spaces involved in the computations and CPU times. $T_{h}$ is the time for the solution of (3.3), $T_{\text {off }}$ is the time needed for the offline stage and $T_{\text {on }}$ the solution time of the online problem.

\begin{tabular}{|c|c|c|c|c|c|c|c|c|c|c|}
\hline & \multicolumn{2}{|c|}{$l=1$} & \multicolumn{2}{|c|}{$l=2$} & \multicolumn{2}{|c|}{$l=3$} & \multicolumn{2}{|c|}{$l=4$} & \multicolumn{2}{|c|}{$l=5$} \\
\hline$N_{\Gamma_{i}}$ & \multicolumn{2}{|c|}{15} & \multicolumn{2}{|c|}{29} & \multicolumn{2}{|c|}{57} & \multicolumn{2}{|c|}{113} & \multicolumn{2}{|c|}{225} \\
\hline$M_{B C}$ & \multicolumn{2}{|c|}{4} & \multicolumn{2}{|c|}{6} & \multirow{2}{*}{\multicolumn{2}{|c|}{$\begin{array}{c}7 \\
5376\end{array}$}} & \multicolumn{2}{|c|}{10} & \multicolumn{2}{|c|}{13} \\
\hline$N_{h}$ & \multicolumn{2}{|c|}{324} & \multicolumn{2}{|c|}{1328} & & & \multirow{2}{*}{\multicolumn{2}{|c|}{$\begin{array}{c}21632 \\
1.4 \mathrm{e}-1\end{array}$}} & \multirow{2}{*}{\multicolumn{2}{|c|}{$\begin{array}{c}86784 \\
6.8 \mathrm{e}-1\end{array}$}} \\
\hline$T_{h}(\mathrm{~s})$ & & -3 & & -3 & \multicolumn{2}{|c|}{$2.7 \mathrm{e}-2$} & & & & \\
\hline Method & $\mathrm{A}$ & $\mathrm{B}$ & $\mathrm{A}$ & $\mathrm{B}$ & $\mathrm{A}$ & $\mathrm{B}$ & $\mathrm{A}$ & $\mathrm{B}$ & $\mathrm{A}$ & $\mathrm{B}$ \\
\hline$N_{R B}$ & 35 & 17 & 57 & 20.5 & 97.5 & 21 & 168 & 27.5 & 293 & 33.5 \\
\hline$T_{\text {off }}$ & $41 \mathrm{~s}$ & $28 \mathrm{~s}$ & $144 \mathrm{~s}$ & $51 \mathrm{~s}$ & $23 \mathrm{~m}$ & $105 \mathrm{~s}$ & $4 \mathrm{~h} 39 \mathrm{~m}$ & $9 \mathrm{~m}$ & $64 \mathrm{~h} 23 \mathrm{~m}$ & $1 \mathrm{~h} 37 \mathrm{~m}$ \\
\hline$N_{D G R B E}$ & 70 & 34 & 114 & 41 & 195 & 42 & 336 & 55 & 586 & 67 \\
\hline$N_{h} / N_{D G R B E}$ & 4.6 & 9.5 & 11.6 & 32.4 & 27.5 & 128 & 64.4 & 393 & 148 & 1295 \\
\hline$T_{\text {on }}(\mathrm{s})$ & $4.7 \mathrm{e}-4$ & $6.6 \mathrm{e}-5$ & $7.0 e-4$ & $7.1 \mathrm{e}-5$ & $2.1 \mathrm{e}-3$ & $7.9 e-5$ & $3.3 e-3$ & $1.0 e-4$ & $7.6 e-3$ & $4.0 e-4$ \\
\hline
\end{tabular}

\subsection{Example 1. Comparison of the two enrichment strategies}

We make a comparison between the two proposed strategies for the construction of the local bases. We tested both strategies on a diffusion reaction problem defined on $\Omega=\Omega_{1} \cup \Omega_{2}$ with $\Omega_{1}=(0,1) \times(0,1)$ and $\Omega_{2}=(1,2) \times(0,1)$. We then consider a parameter $\boldsymbol{\mu}=\left(\mu_{1}, \ldots, \mu_{4}\right)$ belonging to $\mathcal{D}=[0.1,10] \times[0,1]^{3} \subset \mathbb{R}^{4}$ and we set $\nu(\boldsymbol{\mu})=\mu_{1}, \sigma \equiv 1$. The right-hand side function $f(\boldsymbol{\mu})$ is chosen such that the exact solution is:

$$
u(\boldsymbol{\mu})=\frac{\mu_{2}}{\mu_{1}} \sin (\pi x) \sin (\pi y)+\frac{\mu_{3}}{\mu_{1}} \frac{1}{2} \sin (2 \pi x) \sin (2 \pi y)+\frac{\mu_{4}}{\mu_{1}} \frac{1}{3} \sin (3 \pi x) \sin (3 \pi y) .
$$

We consider a sequence of uniform refinements $\mathcal{T}_{h_{l}}, l=2, \ldots, 5$, of a given initial grid $\mathcal{T}_{h_{1}}$, with granularity $h_{1}=$ 0.1 , such that $h_{l}=\frac{h_{l-1}}{2}, l=2, \ldots, 5$. In Table 1 we report the dimensions of the spaces involved in the computations and the corresponding CPU times. Here we have denoted with $M_{B C}$ the number of Legendre polynomials considered on each interface, with $N_{h}$ the dimension of the fine FE space upon which the reduced bases are built, with $N_{R B}$ average number of local basis on each subdomain and with $N_{D G R B E}$ the dimension of the whole DGRBE space. From the results reported in Table 1, it is evident that Method A produces larger DGRBE spaces and thus is more expensive than Method B. In Figures 1 and 2 we show the (relative) approximation error computed in the energy and the $\mathrm{L}^{2}$ norms, respectively, of the enriched DGRBE method and we compare it with the corresponding fine-grid FE solution (relative) approximation error. The errors are computed with respect to $u(\boldsymbol{\mu})$ and represent an average on a sample of 24 parameter values. We can observe that Method A exhibits the same approximation properties than those provided by the FE method. Indeed the error curves reported in Figure 1a are almost overlapped. The same is observed for Method B, $c f$. Figure 1b. In Figure 3 we show the DGRBE approximation relative error, with respect to the fine-grid approximation (3.3), as a function of the number of local basis used (for $l=3$ ). We observe that Method A shows a regular decay until it reaches a minimum value which is actually higher than the local RB tolerance, as expected by the analysis. As for Method B, we observe a faster, but less regular, decay. Suitable strategies to improve this convergence feature of Method B are currently under investigation. We note also that the minimum value is higher than the one reached by Method A. This is due to our choice of the number $M_{B C}$ of Legendre polynomials.

From these results we can conclude that the a priori error estimates given by Theorem 3.4 are not sharp. This is due to the fact that the element $z^{R B}(\boldsymbol{\mu})$ considered to build the upper bound does not provide the actual "best approximation" of the continuous solution $u(\boldsymbol{\mu})$ in $V^{R B}$. Indeed, there are cases in which the estimate is sharp for $\left\|u(\boldsymbol{\mu})-z^{R B}(\boldsymbol{\mu})\right\|_{D G}$ but not for the DGRBE approximation error. We consider in particular the estimate of the interface jump component of the Method B error, given by (3.16), which yields the dependence 


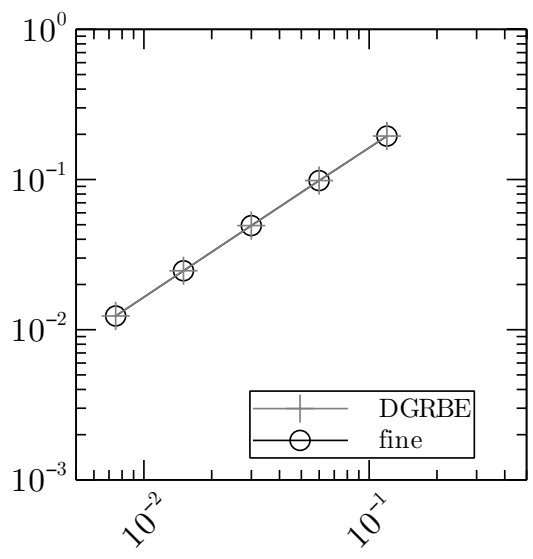

(a) Method A

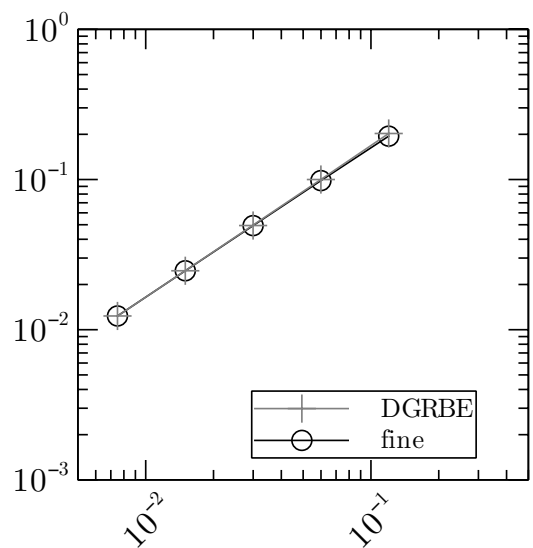

(b) Method B

Figure 1. Example 1. Relative approximation error computed in the energy norm versus $h$, Method A (left) and Method B (right).

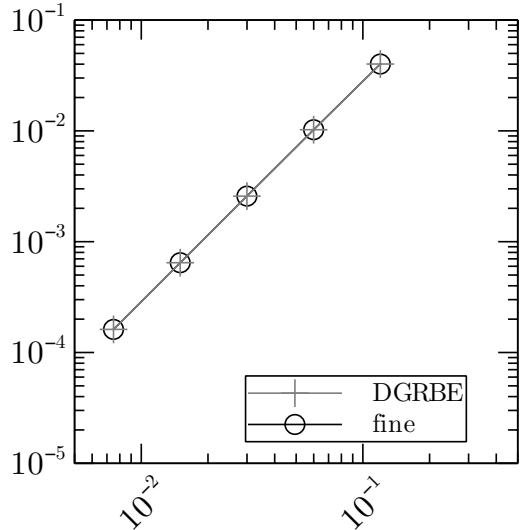

(a) Method A

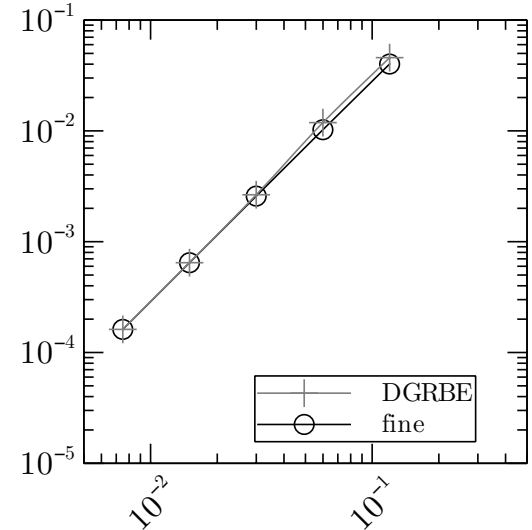

(b) Method B

Figure 2. Example 1. Relative approximation error computed in the $\mathrm{L}^{2}$ norm versus $h$, Method A (left) and Method B (right).

on $h^{-1 / 2}$ of the global estimate. In Figure 4 (left) we show the behaviour of the quantities

$$
\sqrt{\frac{\gamma}{h}}\left\|\llbracket u(\boldsymbol{\mu})-u^{R B}(\boldsymbol{\mu}) \rrbracket\right\|_{\mathrm{L}^{2}\left(\Gamma_{12}\right)} \quad \text { and } \quad \sqrt{\frac{\gamma}{h}}\left\|\llbracket u(\boldsymbol{\mu})-z^{R B}(\boldsymbol{\mu}) \rrbracket\right\|_{L^{2}\left(\Gamma_{12}\right)}
$$

as functions of $h$, for a particular value of $\boldsymbol{\mu}$, using $M_{B C}=3$ Legendre polynomials during the offline stage. From these results we clearly observe that the second quantity in (5.1) scales as $h^{-1 / 2}$, and therefore the estimate given in (3.16) seems to be sharp. Finally, as anticipated in Remark 3.6, we have performed a numerical study of the dual norm of the functional $\mathcal{I}_{i}^{m}$ of Method A (evaluated by computing the norm of theirs $\mathrm{H}^{1}$ Riesz representatives in $V_{h, 1}$ ). In Figure 4 (right) we observe that the maximum value of $\left\|\mathcal{I}_{i}^{m}\right\|_{V_{h, 1}^{\prime}}$ decays as $h$, therefore the constant $\Phi_{\mu}^{A, i}$ defined Lemma 3.2 is independent of $h$. 


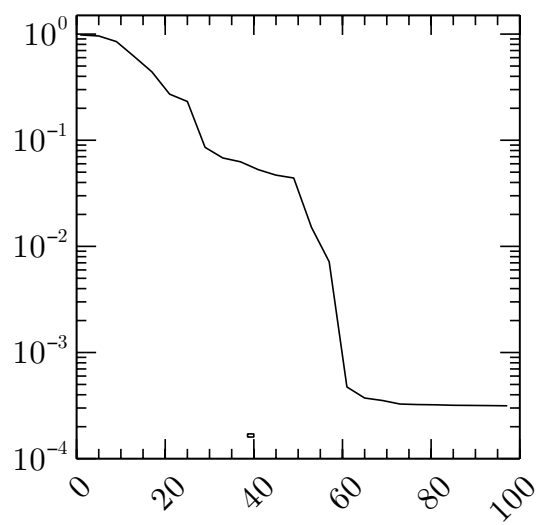

(a) Method A

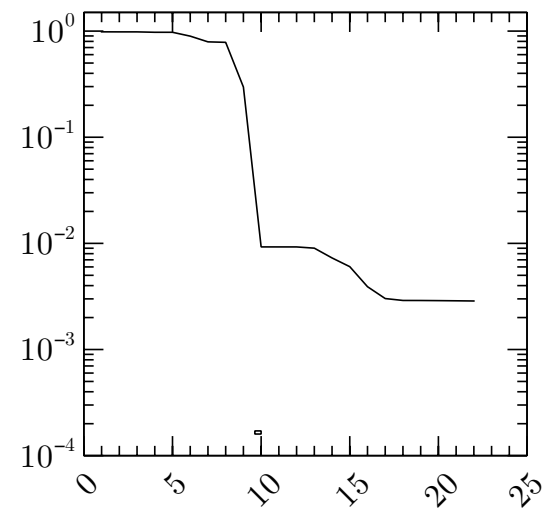

(b) Method B

FiguRE 3. Example 1. Relative approximation error, with respect to the fine-grid solution (3.3), of the two different enrichment methods versus the number of local basis. Here $l=3$.
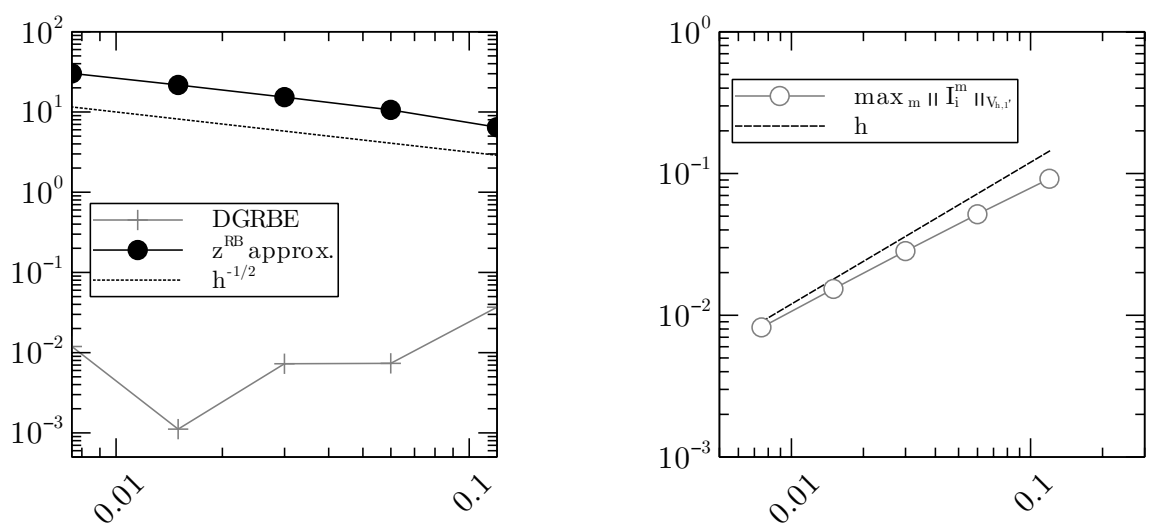

FiguRE 4. Example 1. Left: $\sqrt{\frac{\gamma}{h}}\left\|\llbracket u(\boldsymbol{\mu})-u^{R B}(\boldsymbol{\mu}) \rrbracket\right\|_{\mathrm{L}^{2}\left(\Gamma_{12}\right)}$ and $\sqrt{\frac{\gamma}{h}}\left\|\llbracket u(\boldsymbol{\mu})-z^{R B}(\boldsymbol{\mu}) \rrbracket\right\|_{\mathrm{L}^{2}\left(\Gamma_{12}\right)}$ versus $h,\left(\boldsymbol{\mu}=(1,0,1,0), M_{B C}=3\right.$, Method B). Right: $\max _{m}\left\|\mathcal{I}_{i}^{m}\right\|_{V_{h, 1}^{\prime}}$ versus $h$ (Method A).

\subsection{Example 2. Several subdomain case}

We tested Method B on a domain composed by many subdomains. We define a global domain of the form $\Omega=(0, S) \times(0, S)$ with $S \in \mathbb{N}$, partitioned into $N_{S}=S^{2}$ subdomains. The exact solution is the same considered in Example 1. We built a Legendre's basis on each non-Dirichlet side of the square-shaped subdomains. In Figure 5 we plot the relative DGRBE approximation error as a function of the fine mesh size, and we compare it with the error given by the fine and the coarse FE approximations. The method does not show a worsening of the performances as the number of subdomain increases.

\subsection{Example 3. Two-level preconditioner}

We tested the two level preconditioner of Section 4 on the problem introduced in the Example 2, see Section 5.2. We chose to use $N_{S}=16,64$ and we applied Method B of Section 2.1 to build the local basis. As for the meshes, we define a coarse mesh $\mathcal{T}_{H}$ and a sequence of uniform refinements $\mathcal{T}_{h_{l}}, l=1, \ldots, 6$, 


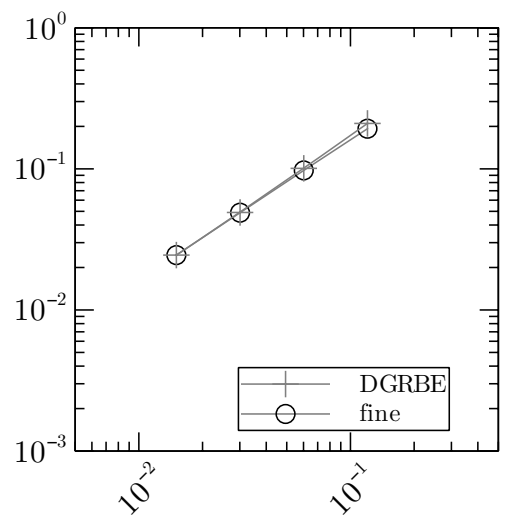

(a) $S=2, N_{S}=4$

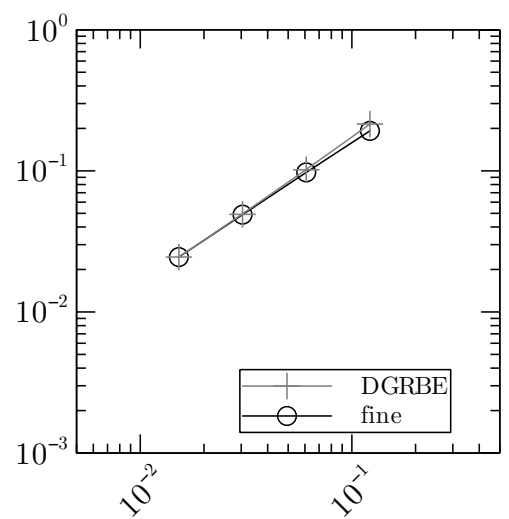

(b) $S=3, N_{S}=9$

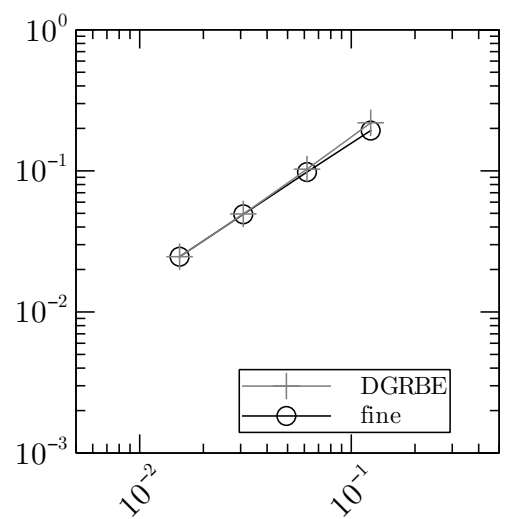

(c) $S=4, N_{S}=16$

Figure 5. Example 2. Comparison of the DGRBE relative approximation error and the FE coarse one, as functions of the fine mesh size $h$.

TABle 2. Example 3, $N_{S}=16$. Condition number of the online system (fine mesh size on the rows, coarse mesh size on the columns) and iteration counts (between parentheses).

(a) Non preconditioned

\begin{tabular}{ccccc}
\hline & No coarse & $H$ & $H / 2$ & $H / 4$ \\
\hline$h$ & $9.68 \mathrm{e} 2(44)$ & $1.94 \mathrm{e} 2(51)$ & $/$ & $/$ \\
$h / 2$ & $1.91 \mathrm{e} 3(98)$ & $4.04 \mathrm{e} 2(100)$ & $5.17 \mathrm{e} 2(109)$ & $/$ \\
$h / 4$ & $3.78 \mathrm{e} 3(131)$ & $8.20 \mathrm{e} 2(135)$ & $1.06 \mathrm{e} 3(152)$ & $1.66 \mathrm{e} 3(163)$ \\
$h / 8$ & $7.50 \mathrm{e} 3(176)$ & $1.65 \mathrm{e} 3(195)$ & $2.15 \mathrm{e} 3(184)$ & $3.40 \mathrm{e} 3(223)$ \\
$h / 16$ & $1.49 \mathrm{e} 4(218)$ & $3.31 \mathrm{e} 3(252)$ & $4.32 \mathrm{e} 3(264)$ & $6.87 \mathrm{e} 3(271)$ \\
\hline
\end{tabular}

(b) Preconditioned

\begin{tabular}{cccc}
\hline & $H$ & $H / 2$ & $H / 4$ \\
\hline$h$ & $11.4(21)$ & $/$ & $/$ \\
$h / 2$ & $25.6(32)$ & $11.6(25)$ & $/$ \\
$h / 4$ & $52.7(41)$ & $23.7(33)$ & $9.87(22)$ \\
$h / 8$ & $104(52)$ & $54.1(43)$ & $21.5(29)$ \\
$h / 16$ & $205(63)$ & $109(53)$ & $44.0(37)$ \\
\hline
\end{tabular}

TABLE 3. Example $3, N_{S}=64$. Condition number of the online system (fine mesh size on the rows, coarse mesh size on the columns) and iteration counts (between parentheses).

(a) Non preconditioned

\begin{tabular}{ccccc}
\hline & No coarse & $H$ & $H / 2$ & $H / 4$ \\
\hline$h$ & $1.73 \mathrm{e} 3(127)$ & $6.04 \mathrm{e} 2(87)$ & $/$ & $/$ \\
$h / 2$ & $2.73 \mathrm{e} 3(230)$ & $1.24 \mathrm{e} 3(157)$ & $1.67 \mathrm{e} 3(181)$ & $/$ \\
$h / 4$ & $5.43 \mathrm{e} 3(304)$ & $2.52 \mathrm{e} 3(207)$ & $3.42 \mathrm{e} 3(215)$ & $5.41 \mathrm{e} 3(222)$ \\
$h / 8$ & $1.08 \mathrm{e} 4(408)$ & $5.08 \mathrm{e} 3(300)$ & $6.93 \mathrm{e} 3(311)$ & $1.11 \mathrm{e} 4(335)$ \\
$h / 16$ & $2.17 \mathrm{e} 4(539)$ & $1.02 \mathrm{e} 4(384)$ & $1.39 \mathrm{e} 4(331)$ & $2.23 \mathrm{e} 4(341)$ \\
\hline
\end{tabular}

\begin{tabular}{cccc}
\hline & $H$ & $H / 2$ & $H / 4$ \\
\hline$h$ & $11.4(21)$ & $/$ & $/$ \\
$h / 2$ & $25.6(33)$ & $11.6(26)$ & $/$ \\
$h / 4$ & $53.0(43)$ & $23.8(33)$ & $9.92(23)$ \\
$h / 8$ & $105(54)$ & $54.2(46)$ & $21.6(31)$ \\
$h / 16$ & $205(63)$ & $109(53)$ & $44.0(37)$ \\
\hline
\end{tabular}

assuming that the restrictions of these meshes to each subdomain are conforming triangulations. We then set

$$
\mathcal{T}_{H}^{\prime}=\mathcal{T}_{h_{1}}, \quad \mathcal{T}_{h_{l}}^{\prime}=\mathcal{T}_{h_{l+1}}, \quad l=1, \ldots, 5, \quad \text { and } \quad \mathcal{T}_{H}^{\prime \prime}=\mathcal{T}_{h_{2}}, \quad \mathcal{T}_{h_{l}}^{\prime}=\mathcal{T}_{h_{l+2}}, \quad l=1, \ldots, 4 .
$$

In Tables 2 and 3 we show the condition number of the non-preconditioned online system and the preconditioned one, for $N_{S}=16$ and $N_{S}=64$, respectively. In brackets we report the numbers of conjugate gradient iterations needed to solve the online system (2.12) (with a tolerance of $10^{-9}$ ). As regards the non-preconditioned case, the values reported are referred to the online matrices associated with the space (2.7) (column "no coarse") 
TABLE 4. Example 3. CPU time (in seconds) for the solution of the online preconditioned system (fine mesh size on the rows, coarse mesh size on the columns) and corresponding number of degrees of freedom, for $N_{S}=64$. The CPU time for the solution of the non-preconditioned system is also reported (within brackets).

(a) CPU time (s)

\begin{tabular}{ccccc}
\hline & No coarse & $H$ & $H / 2$ & $H / 4$ \\
\hline$h$ & $/(0.037)$ & $0.049(0.049)$ & $/$ & $/$ \\
$h / 2$ & $/(0.249)$ & $0.166(0.228)$ & $0.166(0.293)$ & $/$ \\
$h / 4$ & $/(0.464)$ & $0.233(0.400)$ & $0.242(0.521)$ & $0.467(0.762)$ \\
$h / 8$ & $/(0.804)$ & $0.324(0.748)$ & $0.360(0.841)$ & $0.360(1.340)$ \\
$h / 16$ & $/(1.670)$ & $0.466(1.300)$ & $0.511(1.210)$ & $0.789(1.730)$ \\
\hline
\end{tabular}

(b) Number of degrees of freedom

\begin{tabular}{ccccc}
\hline & No coarse & $H$ & $H / 2$ & $H / 4$ \\
\hline$h$ & 1444 & 1444 & $/$ & $/$ \\
$h / 2$ & 2506 & 3043 & 3851 & $/$ \\
$h / 4$ & 3019 & 3550 & 4506 & 7871 \\
$h / 8$ & 3483 & 3998 & 4903 & 8324 \\
$h / 16$ & 4321 & 4809 & 5747 & 9121 \\
\hline
\end{tabular}

TABLE 5. Example 3. Comparison of the preconditioned reduced scheme (with coarse mesh size $H \approx 0.5$ ) and of the fine-grid approximation of the problem. $T_{h}$ is the time needed for the solution of (3.3), $T_{\text {off }}$ is the time required for the local offline stage on a single subdomain, and $T_{\mathrm{on}}$ is the solution time of the online problem.

\begin{tabular}{ccccccc}
\hline Mesh size & $N_{h}$ & $T_{h}$ & Average $T_{\text {off }}$ & $M_{B C}$ & $N_{D G R B E}$ & $T_{\text {on }}$ \\
\hline$h / 8 \approx 0.06$ & 68644 & $7.280 \mathrm{~s}$ & $1 \mathrm{~m} 40 \mathrm{~s}$ & 5 & 3998 & $0.324 \mathrm{~s}$ \\
$h / 16 \approx 0.03$ & 268324 & $8.310 \mathrm{~s}$ & $5 \mathrm{~m}$ & 7 & 4809 & $0.466 \mathrm{~s}$ \\
\hline
\end{tabular}

and with the spaces (4.2) for different values of the coarse mesh size (columns " $H$ ", " $H / 2$ " and " $H / 4$ "). The condition number of the non-preconditioned matrix has been evaluated by explicitly computing the extremal eigenvalues. Differently, the condition number of the preconditioned system has been computed as in [2], by exploiting the connections between the Lanczos technique and the Preconditioned Conjugate Gradient (PCG) method, as presented in detail in [18]. We observe that the condition number of the preconditioned system scales as expected by Proposition 4.5. We observe also the condition number of the preconditioned system and thus the number of PCG iterations are independent of the number of subdomains. In our tests we used an initial coarse mesh size $H \approx 0.5$. In Table 4 a we show the CPU times for the solution of the preconditioned online system and the non-preconditioned one (within brackets), for $N_{S}=64$. The speed-up ensured by the preconditioner represents a trade-off between the reduction of the number of iterations from one hand, and the increase of the number of degrees of freedom (see Tabs. 4 and 5) that are brought by the coarse space. We point out that the reported CPU times refer to a serial (not parallel) implementation of the preconditioner. For the sake of completeness, in Table $4 \mathrm{~b}$ we compare the performance of the preconditioned reduced scheme (using coarse mesh size $H$ ) with the PCG solution of the fine problem (3.3), for very refined fine-grids, i.e., the ones with granularity $h / 8 \approx 0.06$ and $h / 16 \approx 0.03$. Here, $T_{h}$ is the elapsed time for the solution of the fine-grid problem, $T_{\text {off }}$ is the time needed to compute a single local basis, $M_{B C}$ is the number of Legendre polynomials considered on each interface, $N_{D G R B E}$ is the DGRBE space dimension, and $T_{\text {on }}$ is the online CPU time. We observe that the reduction of the speed-up with respect to the 2 subdomain case ( $c f$. Example 1$)$ is partly due to the increased number of interfaces which requires a larger number of basis.

\section{Conclusions}

We have introduced the DGRBE method and carried out its convergence analysis. In particular we have proven the well-posedness of the method and we have shown that the DGRBE approximation error scales as the size of the fine mesh on which the local bases are built, provided we use properly defined local problems to build the local bases and set a sufficiently small tolerance in the local Greedy procedures. We have presented two possible methods to generate the local bases. We have finally proposed a possible preconditioner for the online 
problem, which exploits the pre-existing decomposition of the domain, but requires a slight modification in the Greedy procedure used to build the local bases. The modified Greedy algorithm is needed to ensure the linear independence between the coarse space and the local bases, which is crucial to guarantee the well-posedness of the method. Through numerical experiments, we have compared the DGRBE approximation with the FE one based on the fine meshes on which the local basis are built. We have shown that the accuracy of both methods is similar, but the former is based on a lower dimensional approximation space. This property holds for any given fine triangulation. We also tested the performances of the proposed preconditioner. Further developments of this work are the study of a posteriori error estimators which provide upper bounds for the approximation error of the reduced solution with respect to the fine-grid one. Moreover, the strategy proposed can be extended to more general problems, e.g. the Stokes problem, in which some stabilization techniques may be needed to guarantee the stability of the method.

\section{Appendix A. Implementation issues}

We now sum up the main ingredients of the DGRBE method, from the implementation point of view.

As regards the offline stage of the DGRBE method, we point out that it can be split into two sub-steps: (i) the local offline stage, where the local reduced bases and the local discrete operators are built; (ii) the global offline stage, where the global DGRBE space and the interface discrete operators are constructed.

We consider now the local offline stage on the subdomain $\Omega_{i}$. With respect to a given $\mathrm{FE}$ basis $\mathcal{B}_{h, i}=$ $\left\{\varphi_{1}^{i}, \ldots, \varphi_{N_{h, i}}^{i}\right\}$ of $V_{h, i}$, given $\widetilde{\boldsymbol{\mu}}=(\boldsymbol{\mu}, \beta) \in \widetilde{\mathcal{D}}$ the matrix form of the reference problem (2.5) reads

$$
\mathbf{A}_{h_{i}}(\boldsymbol{\mu}) \mathbf{u}_{h_{i}}(\boldsymbol{\mu})=\mathbf{F}_{h_{i}}(\boldsymbol{\mu})+\mathbf{I}_{h_{i}}(\beta) .
$$

As usual in the RB context, we assume that the local operators depends "affinely" on the parameter $[32,34]$, i.e.,

$$
\mathbf{A}_{h_{i}}(\boldsymbol{\mu})=\sum_{q=1}^{Q_{A_{i}}} \Theta_{A_{i}}^{q}(\boldsymbol{\mu}) \mathbf{A}_{h, i}^{q}, \quad \mathbf{F}_{h_{i}}(\boldsymbol{\mu})=\sum_{q=1}^{Q_{F_{i}}} \Theta_{F_{i}}^{q}(\boldsymbol{\mu}) \mathbf{F}_{h, i}^{q}, \quad \mathbf{I}_{h_{i}}(\beta)=\sum_{q=1}^{Q_{\mathcal{I}_{i}}} \Theta_{\mathcal{I}_{i}}^{q}(\beta) \mathbf{I}_{h, i}^{q},
$$

where $\mathbf{A}_{h, i}^{q}, \mathbf{F}_{h, i}^{q}$ and $\mathbf{I}_{h, i}^{q}$ are parameter independent arrays, while $\Theta_{A_{i}}^{q}, \Theta_{F_{i}}^{q}$ and $\Theta_{\mathcal{I}_{i}}^{q}$ are real valued functions of the parameter $\boldsymbol{\mu} \in \mathcal{D}$ and $\beta \in \mathcal{D}_{B C}$. In order to recover such "affine" decomposition of the operators, suitable empirical interpolation techniques may be needed [8]. We denote with $\mathcal{B}_{R B, i}=\left\{\zeta_{1}^{i}, \ldots, \zeta_{N_{i}}^{i}\right\} \subseteq V_{h, i}$ the basis of the space $V_{i}^{R B}$ produced by the Greedy's algorithm, i.e., the local reduced basis. We denote with $\mathbf{Z}_{R B, i}$ the matrices whose columns are the coefficients of the expansion of the elements of $\mathcal{B}_{R B, i}$ with respect to the fine basis $\mathcal{B}_{h, i}$. In the local offline stage the following matrices and vectors are then built and stored:

$$
\begin{array}{ll}
\mathbf{A}_{R B, i, i}^{q}=\mathbf{Z}_{R B, i}^{\mathrm{T}} \mathbf{A}_{h, i}^{q} \mathbf{Z}_{R B, i} & \forall q=1, \ldots, Q_{A_{i}}, \\
\mathbf{F}_{R B, i}^{q}=\mathbf{Z}_{R B, i}^{\mathrm{T}} \mathbf{F}_{h, i}^{q}, & \forall q=1, \ldots, Q_{F_{i}}, \\
\mathbf{I}_{R B, i}^{q}=\mathbf{Z}_{R B, i}^{\mathrm{T}} \mathbf{I}_{h, i}^{q}, & \forall q=1, \ldots, Q_{\mathcal{I}_{i}} .
\end{array}
$$

As for the global offline stage, we start by considering the matrices associated to the interface terms through the bases $\mathcal{B}_{h, i}$ and $\mathcal{B}_{h, j}$. Setting $\bar{\Gamma}_{i j}(\boldsymbol{\mu})=\overline{\partial \Omega}_{i}(\boldsymbol{\mu}) \cap \overline{\partial \Omega}_{j}(\boldsymbol{\mu})$, as regards the jump term we have

$$
\left(\mathbf{C}_{r, r^{\prime}}(\boldsymbol{\mu})\right)_{p q}=\frac{\nu(\boldsymbol{\mu}) \gamma}{h} \int_{\Gamma_{i j}(\boldsymbol{\mu})} \varphi_{q}^{r^{\prime}} \varphi_{p}^{r} \mathrm{~d} s \quad \forall p=1, \ldots, N_{h, r^{\prime}} \quad \forall q=1, \ldots, N_{h, r} \quad \forall r, r^{\prime} \in\{i, j\} .
$$

As for the derivative consistency term, we define

$$
\left(\mathbf{D}_{r, r^{\prime}}(\boldsymbol{\mu})\right)_{p q}=\int_{\Gamma_{i j}(\boldsymbol{\mu})} \frac{1}{2} \nu(\boldsymbol{\mu})\left(\nabla \varphi_{q}^{r^{\prime}} \cdot \boldsymbol{n}_{r^{\prime}}\right) \varphi_{p}^{r} \mathrm{~d} s \quad \forall p=1, \ldots, N_{h, r^{\prime}} \quad \forall q=1, \ldots, N_{h, r} \quad \forall r, r^{\prime} \in\{i, j\},
$$


where $\boldsymbol{n}_{r}$ is the normal unit vector of $\Gamma_{i j}(\boldsymbol{\mu})$, exiting from $\Omega_{r}$. We assume that also these interface matrices admit an affine decomposition as in (A.1), i.e.,

$$
\mathbf{C}_{r, r^{\prime}}(\boldsymbol{\mu})=\sum_{q=1}^{Q_{C_{r, r^{\prime}}}} \Theta_{C_{r, r^{\prime}}}^{q}(\boldsymbol{\mu}) \mathbf{C}_{r, r^{\prime}}^{q}, \quad \mathbf{D}_{r, r^{\prime}}(\boldsymbol{\mu})=\sum_{q=1}^{Q_{D_{r, r^{\prime}}}} \Theta_{D_{r, r^{\prime}}}^{q}(\boldsymbol{\mu}) \mathbf{D}_{r, r^{\prime}}^{q}
$$

for suitably chosen parameter independent matrices $\mathbf{C}_{r, r^{\prime}}^{q}, \mathbf{D}_{r, r^{\prime}}^{q}$ and real valued functions $\Theta_{C_{r, r^{\prime}}}^{q}, \Theta_{D_{r, r^{\prime}}}^{q}$ of $\boldsymbol{\mu}$.

In the global offline stage we build and store the following matrices:

$$
\begin{array}{lll}
\mathbf{C}_{R B, r, r^{\prime}}^{q}=S\left(r, r^{\prime}\right) \mathbf{Z}_{R B, r}^{\mathrm{T}} \mathbf{C}_{r, r^{\prime}}^{q} \mathbf{Z}_{R B, r^{\prime}} & \forall q=1, \ldots, Q_{C_{r, r^{\prime}}} & \forall r, r^{\prime} \in\{i, j\}, \\
\mathbf{D}_{R B, r, r^{\prime}}^{q}=S\left(r, r^{\prime}\right) \mathbf{Z}_{R B, r}^{\mathrm{T}} \mathbf{D}_{r, r^{\prime}}^{q} \mathbf{Z}_{R B, r^{\prime}} & \forall q=1, \ldots, Q_{D_{r, r^{\prime}}} & \forall r, r^{\prime} \in\{i, j\},
\end{array}
$$

where $S\left(r, r^{\prime}\right)=1$ if $r=r^{\prime}, S\left(r, r^{\prime}\right)=-1$ otherwise, and $Q_{C_{r, r^{\prime}}}, Q_{D_{r, r^{\prime}}}$ are the numbers of affine terms of $\mathbf{C}_{r, r^{\prime}}$ and $\mathbf{D}_{r, r^{\prime}}$, respectively. These matrices have to be built for each interface.

During the online stage, the matrices built and stored during the offline stage have to be properly assembled exploiting the affine decomposition property, for a given value of the parameter $\tilde{\boldsymbol{\mu}} \in \mathcal{D}$. First of all the arrays $\mathbf{A}_{R B, i, j}(\boldsymbol{\mu}), \mathbf{C}_{R B, i, j}(\boldsymbol{\mu}), \mathbf{D}_{R B, i, j}(\boldsymbol{\mu})$ and $\mathbf{F}_{R B, i}(\boldsymbol{\mu})$ have to be built, exploiting the affine decomposition (which follows from the fine-grid operators affine decomposition (A.1) and (A.2)), by summing the previously stored quantities. In order to simplify the exposition, if $\bar{\Omega}_{i} \cap \bar{\Omega}_{j}=\emptyset$, we assume that $\mathbf{C}_{R B, i, j}(\boldsymbol{\mu})$ and $\mathbf{D}_{R B, i, j}(\boldsymbol{\mu})$ are null matrices of dimension $N_{i} \times N_{j}$. Moreover, if $i \neq j$, we assume that $\mathbf{A}_{R B, i, j}(\boldsymbol{\mu})$ is the null matrix of dimension $N_{i} \times N_{j}$. For the sake of notation, we now omit the parameter dependence of matrices and vectors. We define:

$$
\mathbf{A}_{\mathrm{On}}=\left(\mathbf{A}_{R B, i, j}\right)_{i, j=1, \ldots, N_{S}}, \quad \mathbf{C}_{\mathrm{On}}=\left(\mathbf{C}_{R B, i, j}\right)_{i, j=1, \ldots, N_{S}}, \quad \mathbf{D}_{\mathrm{On}}=\left(\mathbf{D}_{R B, i, j}\right)_{i, j=1, \ldots, N_{S}} .
$$

The matrix associated to the online problem (2.12) is then: $\mathbf{A}_{R B}=\mathbf{A}_{\mathrm{On}}+\mathbf{C}_{\mathrm{On}}+\mathbf{D}_{\mathrm{On}}+\mathbf{D}_{\text {On }}^{\mathrm{T}}$. We finally define $\mathbf{u}_{R B}=\left(\mathbf{u}_{N_{i}}\right)_{i=1, \ldots, N_{S}}$, and $\mathbf{F}_{R B}=\left(\mathbf{F}_{N_{i}}\right)_{i=1, \ldots, N_{S}}$. The algebraic system associated with the online problem (2.12) is then

$$
\mathbf{A}_{R B} \mathbf{u}_{R B}=\mathbf{F}_{R B} .
$$

\section{Appendix B. Construction of the local BAsis For THE TWO-LEVel PRECONDITIONER}

To make sure that the local reduced spaces $V_{N_{i}, i}$ and the coarse ones $V_{H, i}$ are in direct sum as assumed in (4.2) the standard implementation of the RB Greedy's algorithm [34] will not serve the purpose. We propose a possible way to build the space $V_{N_{i}, i}$, which is described in detail in the pseudo-algorithm below (Algorithm 1). It is a proper modification of the algorithm shown in $[32,34]$. We consider local a posteriori error estimators $\Delta_{H, N_{i}}^{k}: \widetilde{\mathcal{D}} \rightarrow R, i=1, \ldots, N_{S}$, based on the dual norm of the residual, similarly to the a posteriori estimator of the standard RB method (cf. [32,34]). The estimator $\Delta_{H, i}^{k}$ satisfies

$$
\frac{\left\|\widetilde{u}_{h, i}(\widetilde{\boldsymbol{\mu}})-u_{H, i}^{k}(\widetilde{\boldsymbol{\mu}})\right\|_{\tilde{\boldsymbol{\mu}}, i}}{\left\|u_{H, i}^{k}(\widetilde{\boldsymbol{\mu}})\right\|_{V_{i}}} \leq \Delta_{H, i}^{k}(\widetilde{\boldsymbol{\mu}}) \quad \forall \widetilde{\boldsymbol{\mu}} \in \mathcal{D},
$$

where $\widetilde{u}_{h, i}(\widetilde{\boldsymbol{\mu}})$ solves $(2.5)$ and $u_{H, i}^{k}(\widetilde{\boldsymbol{\mu}}) \in V_{H, i}^{k}$ is such that

$$
A_{i}\left(u_{H, i}^{k}(\widetilde{\boldsymbol{\mu}}), v_{H, i}^{k} ; \widetilde{\boldsymbol{\mu}}\right)=F_{i}\left(v_{H, i}^{k} ; \widetilde{\boldsymbol{\mu}}\right)+\left\langle\mathcal{I}_{i}^{\beta},\left.v_{H, i}^{k}\right|_{\Gamma_{i}}\right\rangle \quad \forall v_{H, i}^{k} \in V_{H, i}^{k},
$$

with $V_{H, i}^{k}=V_{H, i} \oplus \operatorname{span}\left\{\widetilde{u}_{h, i}\left(\widetilde{\boldsymbol{\mu}}_{i}^{1}\right), \ldots, \widetilde{u}_{h, i}\left(\widetilde{\boldsymbol{\mu}}_{i}^{k}\right)\right\}$. Note that $V_{H, i}^{0}=V_{H, i}$. It holds that

$$
\Delta_{H, i}^{k}(\widetilde{\boldsymbol{\mu}})=0 \Longleftrightarrow \widetilde{u}_{h, i}(\widetilde{\boldsymbol{\mu}}) \in V_{H, i}^{k},
$$


cf. [34]. Thanks to (B.2), if the parameter value $\widetilde{\boldsymbol{\mu}}_{i}^{k+1}$ picked up at the $k$ th step of the Greedy's algorithm is such that $\Delta_{H, i}^{k}\left(\widetilde{\boldsymbol{\mu}}_{i}^{k+1}\right)>0$, then $\widetilde{u}_{h, i}\left(\widetilde{\boldsymbol{\mu}}_{i}^{k+1}\right)$ is independent of $V_{H, i}^{k}$ and, in particular of $V_{H, i}$.

At each step of our Greedy's algorithm, we ensure also some orthogonality properties on the local basis. This is useful to control the conditioning of the linear system associated with problem (B.1), to be solved many times during the Greedy's procedure.

Algorithm 1 (Greedy's algorithm for the $i$ th local problem).

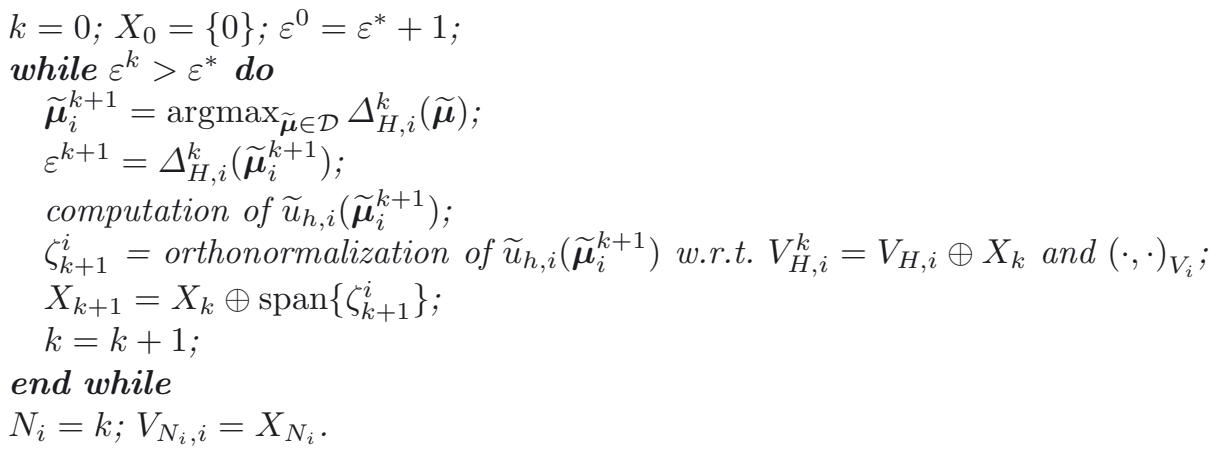

\section{Appendix C. Proof of inequality (3.12)}

The proof of inequality (3.12) follows from standard arguments, for the sake of completeness we sketch it.

Proposition C.1. It holds that

$$
\frac{1}{h} \sum_{\left\{i, j: \bar{\Omega}_{i} \cap \bar{\Omega}_{j} \neq \emptyset\right\}}\left\|\llbracket v_{h} \rrbracket\right\|_{\mathrm{L}^{2}\left(\Gamma_{i j}\right)}^{2} \lesssim \frac{1}{h} \sum_{i=1}^{N_{S}}\left\|v_{h, i}\right\|_{\mathrm{H}^{1}\left(\Omega_{i}\right)}^{2} \quad \forall v_{h} \in V_{h} .
$$

where $h$ is the size of the mesh associated with $V_{h}$.

Proof. We consider the interface $\Gamma_{i j}=\bar{\Omega}_{i} \cap \bar{\Omega}_{j}$. We introduce the lifting operators associated with each interface edge $e$ of $\mathcal{T}_{h}$ belonging to $\Gamma_{i j}$

$$
\begin{aligned}
& r_{e}:\left(\mathrm{H}^{1}\left(\Omega_{i}\right) \oplus \mathrm{H}^{1}\left(\Omega_{j}\right)\right) \rightarrow\left[V_{h}\right]^{2} \\
& \psi \mapsto r_{e}(\psi)
\end{aligned}
$$

where $r_{e}(\psi)$ is such that

$$
\int_{\Omega} r_{e}(\psi) \cdot \tau_{h} \mathrm{~d} x=-\int_{e}\left\{\tau_{h}\right\} \cdot \llbracket \psi \rrbracket \quad \forall \tau_{h} \in\left[V_{h}\right]^{2} .
$$

We know from ([11], Lem. 2) that for $\psi_{h} \in V_{h}$ then

$$
\frac{1}{h}\left\|\llbracket \psi_{h} \rrbracket\right\|_{\mathrm{L}^{2}(e)}^{2} \lesssim\left\|r_{e}\left(\psi_{h}\right)\right\|_{\mathrm{L}^{2}(\Omega)}^{2},
$$

thus we have that

$$
\frac{1}{h}\left\|\llbracket \psi_{h} \rrbracket\right\|_{\mathrm{L}^{2}\left(\Gamma_{i j}\right)}^{2} \lesssim \sum_{e \in \Gamma_{i j}}\left\|r_{e}\left(\psi_{h}\right)\right\|_{\mathrm{L}^{2}(\Omega)}^{2} .
$$

Now, we bound the $\mathrm{L}^{2}$ norm of $r_{e}\left(\psi_{h}\right)$ with the $\mathrm{H}^{1}$ norm of $\psi_{h}$, with an argument similar to that used in ([11], Lem. 2). From (C.1) we have, for each $e \in \Gamma_{12}$,

$$
\left\|r_{e}\left(\psi_{h}\right)\right\|_{\mathrm{L}^{2}(\Omega)}^{2}=-\int_{e}\left\{r_{e}\left(\psi_{h}\right)\right\} \cdot \llbracket \psi_{h} \rrbracket \leq\left\|\llbracket \psi_{h} \rrbracket\right\|_{\mathrm{L}^{2}(e)}\left\|\left\{r_{e}\left(\psi_{h}\right)\right\}\right\|_{\mathrm{L}^{2}(e)} .
$$


Recalling the standard inverse inequality, cf. [31],

$$
\left|v_{h, i}\right|_{\mathrm{H}^{1}\left(\Omega_{i}\right)} \lesssim h^{-1}\left\|v_{h, i}\right\|_{\mathrm{L}^{2}\left(\Omega_{i}\right)} \quad \forall v_{h, i} \in V_{h, i}
$$

and summing over the interface edges we get:

$$
\begin{aligned}
\sum_{e \in \Gamma_{i j}}\left\|r_{e}\left(\psi_{h}\right)\right\|_{\mathrm{L}^{2}(\Omega)}^{2} & \leq \sum_{e \in \Gamma_{i j}}\left\|\llbracket \psi_{h} \rrbracket\right\|_{\mathrm{L}^{2}(e)}\left\|\left\{r_{e}\left(\psi_{h}\right)\right\}\right\|_{\mathrm{L}^{2}(e)} \\
& \leq\left\|\llbracket \psi_{h} \rrbracket\right\|_{\mathrm{L}^{2}\left(\Gamma_{i j}\right)} \sum_{e \in \Gamma_{i j}}\left\|\left\{r_{e}\left(\psi_{h}\right)\right\}\right\|_{\mathrm{L}^{2}(e)} \\
& \stackrel{(3.7)}{\lesssim}\left[\sum_{k \in\{i, j\}}\left\|\psi_{h}\right\|_{\mathrm{H}^{1}\left(\Omega_{k}\right)}\right] \sum_{e \in \Gamma_{i j}}\left\|\left\{r_{e}\left(\psi_{h}\right)\right\}\right\|_{\mathrm{L}^{2}(e)} \\
& \stackrel{(3.11)}{\lesssim}\left[\sum_{k \in\{i, j\}}\left\|\psi_{h}\right\|_{\mathrm{H}^{1}\left(\Omega_{k}\right)}\right] \sum_{e \in \Gamma_{i j}}\left[h^{-1}\left\|r_{e}\left(\psi_{h}\right)\right\|_{\mathrm{L}^{2}(\Omega)}+h \sum_{k \in\{i, j\}}\left|r_{e}\left(\psi_{h}\right)\right|_{\mathrm{H}^{1}\left(\Omega_{k}\right)}\right]^{1 / 2} \\
& (\mathrm{C} .3)\left[\sum_{k \in\{i, j\}}\left\|\psi_{h}\right\|_{\mathrm{H}^{1}\left(\Omega_{k}\right)}\right] \sum_{e \in \Gamma_{i j}}\left[h^{-1}\left\|r_{e}\left(\psi_{h}\right)\right\|_{\mathrm{L}^{2}(\Omega)}\right]^{1 / 2} .
\end{aligned}
$$

We obtain

$$
\sum_{e \in \Gamma_{i j}}\left\|r_{e}\left(\psi_{h}\right)\right\|_{\mathrm{L}^{2}(\Omega)} \lesssim h^{-1 / 2}\left[\sum_{k \in\{i, j\}}\left\|\psi_{h}\right\|_{\mathrm{H}^{1}\left(\Omega_{i}\right)}\right] .
$$

Then, using (C.2) and summing over all the interfaces, we can conclude.

\section{REFERENCES}

[1] F. Albrecht, B. Haasdonk, S. Kaulmann and M. Ohlberger, The localized reduced basis multiscale method. In vol. 1 of Algoritmy 2012 - Proc. of contributed papers and posters, edited by A. Handlovičová, Z. Minarechová and D. Devčovič. Publishing House of STU (2012) 393-403.

[2] P.F. Antonietti and B. Ayuso, Schwarz domain decomposition preconditioners for discontinuous Galerkin approximations of elliptic problems: Non-overlapping case. ESAIM: M2AN 41 (2007) 21-54.

[3] P.F. Antonietti and P. Houston, A class of domain decomposition preconditioners for hp-discontinuous Galerkin finite element methods. J. Sci. Comput. 46 (2011) 124-149.

[4] P.F. Antonietti, S. Giani and P. Houston, Domain decomposition preconditioners for discontinuous Galerkin methods for elliptic problems on complicated domains. J. Sci. Comput. 60 (2014) 203-227.

[5] P.F. Antonietti, A. Manzoni, P Pacciarini and A. Quarteroni, A posteriori error control for discontinuous Galerkin reduced basis element approximations of parametrized elliptic pdes. In preparation (2016)

[6] D.N. Arnold, An interior penalty finite element method with discontinuous elements. SIAM J. Numer. Anal. 19 (1982) $742-760$.

[7] D.N. Arnold, F. Brezzi, B. Cockburn and L.D. Marini, Unified analysis of discontinuous Galerkin methods for elliptic problems. SIAM J. Numer. Anal. 39 (2002) 1749-1779.

[8] M. Barrault, Y. Maday, N.C. Nguyen and A.T. Patera, An "empirical interpolation" method: application to efficient reducedbasis discretization of partial differential equations. C. R. Math. Acad. Sci. Paris 339 (2004) 667-672.

[9] J.H. Bramble and J. Xu, Some estimates for a weighted $L^{2}$ projection. Math. Comput. 56 (1991) 463-476.

[10] H. Brezis, Functional analysis, Sobolev spaces and partial differential equations. Universitext. Springer, New York (2011).

[11] F. Brezzi, M. Manzini, M. Marini, P. Pietra and A. Russo, Discontinuous finite elements for diffusion problems. In Francesco Brioschi (1824-1897) Convegno di Studi Matematici, October 22-23, 1997 Ist. Lomb. Acc. Sc. Lett. Incontro di studio N. 16 (1999) 197-217.

[12] C. Canuto and A. Quarteroni, Approximation results for orthogonal polynomials in Sobolev spaces. Math. Comput. 38 (1982) $67-86$.

[13] Y. Chen, J.S. Hesthaven and Y. Maday, A Seamless Reduced Basis Element Method for 2D Maxwell's Problem: An Introduction. In Spectral and High Order Methods for Partial Differential Equations. Springer (2011) 141-152. 
[14] Y. Efendiev, J. Galvis, R. Lazarov, M. Moon and M. Sarkis, Generalized multiscale finite element method. Symmetric interior penalty coupling. J. Comput. Phys. 255 (2013) 1-15.

[15] J.L. Eftang and A.T. Patera, Port reduction in parametrized component static condensation: Approximation and a posteriori error estimation. Int. J. Numer. Methods Eng. 96 (2013) 269-302.

[16] J.L. Eftang, A.T. Patera and E.M. Rønquist, An " $h p$ " certified reduced basis method for parametrized elliptic partial differential equations. SIAM J. Sci. Comput. 32 (2010) 3170-3200.

[17] K. Gao, E. Chung, R. Gibson, S. Fu and Y. Efendiev, Generalized Multiscale Finite-Element Method (GMsFEM) for elastic wave propagation in heterogeneous, anisotropic media. Available at http://arxiv.org/abs/1409.3550 (2014).

[18] G.H. Golub and C.F. Van Loan, Matrix computations. Johns Hopkins Studies in the Mathematical Sciences, 4th edition. Johns Hopkins University Press, Baltimore, MD (2013).

[19] D.B.P. Huynh, D.J. Knezevic and A.T. Patera, A static condensation Reduced Basis Element method : approximation and a posteriori error estimation. ESAIM: M2AN 47 (2013) 213-251.

[20] D.B.P. Huynh, D.J. Knezevic and A.T. Patera, A static condensation Reduced Basis Element method: Complex problems. Comput. Methods Appl. Mech. Eng. 259 (2013) 197-216.

[21] D.B.P. Huynh, N.C. Nguyen, A.T. Patera and G. Rozza, Rapid reliable solution of the parametrized partial differential equations of continuum mechanics and transport. Available at: http://augustine.mit.edu (2008).

[22] L. Iapichino, Reduced Basis Methods for the Solution of Parametrized PDEs in Repetitive and Complex Networks with Application to CFD. Ph.D. thesis, EPF Lausanne (2012).

[23] L. Iapichino, A. Quarteroni and G. Rozza, Reduced basis method and domain decomposition for elliptic problems in networks and complex parametrized geometries. Comput. Math. Appl. 71 (2016) 408-430.

[24] L. Iapichino, A. Quarteroni and G. Rozza, A reduced basis hybrid method for the coupling of parametrized domains represented by fluidic networks. Comput. Methods Appl. Mech. Eng. 221-222 (2012) 63-82.

[25] S. Kaulmann, M. Ohlberger and B. Haasdonk, A new local reduced basis discontinuous Galerkin approach for heterogeneous multiscale problems. C. R. Math. Acad. Sci. Paris 349 (2011) 1233-1238.

[26] A.E. Løvgren, Y. Maday and E.M. Rønquist, A reduced basis element method for the steady Stokes problem. ESAIM: M2AN 40 (2006) 529-552

[27] A.E. Løvgren, Y. Maday and E.M. Rønquist. A reduced basis element method for the steady Stokes problem: Application to hierarchical flow systems. Model. Identif. Control 27 (2006) 79-94.

[28] Y. Maday and E.M. Rønquist, A Reduced-Basis Element method. J. Sci. Comput. 17 (2002) 447-459.

[29] Y. Maday and E.M. Rønquist, The reduced basis element method: Application to a thermal fin problem. SIAM J. Sci. Comput. 26 (2005) 240-258.

[30] A.T. Patera and G. Rozza, Reduced Basis Approximation and A Posteriori Error Estimation for Parametrized Partial Differential Equations. Version 1.0, Copyright MIT 2006-2007, to appear in (tentative rubric) MIT Pappalardo Graduate Monographs in Mechanical Engineering. Available at: http://augustine.mit.edu (2006).

[31] A. Quarteroni, Numerical models for differential problems. Vol. 8 of MS\&A. Model. Simul. Appl. 2nd edition. Springer, Milan (2014).

[32] A. Quarteroni, G. Rozza and A. Manzoni, Certified reduced basis approximation for parametrized partial differential equations and applications. J. Math. Ind. 1 (2011) 3.

[33] A. Quarteroni, A. Manzoni and F. Negri, Reduced Basis Methods for Partial Differential Equations. An Introduction (2016).

[34] G. Rozza, D.B.P. Huynh and A.T. Patera, Reduced basis approximation and a posteriori error estimation for affinely parametrized elliptic coercive partial differential equations: application to transport and continuum mechanics. Arch. Comput. Methods Eng. State of the Art Reviews 15 (2008) 229-275.

[35] R. Stenberg, Mortaring by a method of J.A. Nitsche, In Computational Mechanics (Buenos Aires, 1998). Centro Internac. Métodos Numér. Ing., Barcelona (1998).

[36] A. Toselli and O. Widlund, Domain Decomposition Methods - Algorithms and Theory. Vol. 34 of Springer Ser. Comput. Math. Springer-Verlag, Berlin (2005).

[37] H. Wang and S. Xiang, On the convergence rates of Legendre approximation. Math. Comput. 81 (2012) 861-877.

[38] M.F. Wheeler, An elliptic collocation-finite element method with interior penalties. SIAM J. Numer. Anal. 15 (1978) $152-161$. 